\title{
Mapping of composite hadrons into elementary hadrons and effective hadronic Hamiltonians
}

\author{
D. HADJIMICHEF ${ }^{a}$, G. KREIN $^{* b}$, S. SZPIGEL $^{c}$ AND J.S. DA VEIGA ${ }^{d}$ \\ ${ }^{a}$ Dep. de Física, Fundação Universidade do Rio Grande, 96201-900 Rio Grande-RS, Brazil \\ ${ }^{b}$ Institut für Kernphysik, Universität Mainz, D-55099 Mainz, Germany \\ ${ }^{c}$ Department of Physics, The Ohio State University, Columbus, OH 43210, USA \\ ${ }^{d}$ Instituto de Fúsica, Universidade de São Paulo, 01452-900 São Paulo-SP, Brazil
}

\begin{abstract}
A mapping technique is used to derive in the context of constituent quark models effective Hamiltonians that involve explicit hadron degrees of freedom. The technique is based on the ideas of mapping between physical and ideal Fock spaces and shares similarities with the quasiparticle method of Weinberg. Starting with the Fock-space representation of single-hadron states, a change of representation is implemented by a unitary transformation such that composites are redescribed by elementary Bose and Fermi field operators in an extended Fock space. When the unitary transformation is applied to the microscopic quark Hamiltonian, effective, hermitian Hamiltonians with a clear physical interpretation are obtained. Applications and comparisons with other composite-particle formalisms of the recent literature are made using the nonrelativistic quark model.
\end{abstract}

PACS: 21.60.Gx, 12.39.-x, 21.30.Fe, 13.75.Lb, 13.75.Cs, 24.85+p,

* Alexander von Humboldt Research Fellow

Permanent address: Instituto de Física Teórica, Universidade Estadual Paulista Rua Pamplona 145, 01405-900 São Paulo-SP, Brazil 


\section{INTRODUCTION}

A great variety of mapping techniques are presented in the literature. In nuclear physics they are used to treat collective oscillations of nuclei. Although available since a long time, there have been attempts only very recently to extend these techniques to hadronic physics, in particular, to constituent quark models. The pioneering work originates from Zhu et al. [1] and Pittel et al. [2]. Zhu et al. use the Composite Particle Representation (CPR) developed by $\mathrm{Wu}$, Feng and collaborators [3] in the context of nuclear physics, for studying the baryon spectrum in the nonrelativistic quark model. Pittel et al. used the Dyson mapping [4] to obtain an effective hadron-hadron interaction from a schematic quark model. A continued effort following this is contained in Refs. [5 8]. Related work is contained in Refs. [9] [14.

This paper considers an approach that was originally developed in the context of atomic physics. It was invented independently by Girardeau [15] and Vorob'ev and Khomkin [16]. The method has been continuously improved throughout the last two decades, and has been used by Girardeau and others in several areas of atomic physics [17,18. Although the method shares several properties with the traditional mappings used in nuclear physics [19], it presents particularities that make it suitable for hadronic problems as we shall discuss shortly ahead. It is based on the ideas of mapping between physical and ideal Fock spaces, and has some similarities with the method of Bohm and Pines [20] to treat collective motions in plasmas. It is a generalization of a transformation employed by S. Tani [21] in 1960 to study single-particle scattering by a potential with a bound state. Girardeau coined the name "Fock-Tani" representation for this method.

In the Fock-Tani representation one starts with the Fock representation of the system using field operators of elementary constituents which satisfy canonical (anti)commutation relations. Composite-particle field operators are linear combinations of the elementary-particle operators and do not generally satisfy canonical (anti)commutation relations. "Ideal" field operators acting on an enlarged Fock space are then introduced in close correspondence with the composite ones. The enlarged Fock space is a graded direct product of the original Fock space and an "ideal state space". The ideal operators correspond to particles with the same quantum numbers of the composites; however, they satisfy by definition canon-

ical (anti)commutation relations. Next, a given unitary transformation, which transforms 
the single composite states into single ideal states, is introduced. When the transformation acts on operators in the subspace of the enlarged Fock space which contains no ideal particles, the transformed operators explicitly express the interactions of composites and constituents. Application of the unitary operator on the microscopic Hamiltonian, or on other hermitian operators expressed in terms of the elementary constituent field operators such as electroweak currents, gives equivalent operators which contain the ideal field operators. The effective Hamiltonians and currents in the new representation are hermitian and have a clear physical interpretation in terms of the processes they describe. Since all field operators in the new representation satisfy canonical (anti)commutation relations, the standard methods of quantum field theory can then be readily applied.

Several characteristics of the Fock-Tani representation make it suitable to the nature of the hadronic problem. It seems to be particularly relevant for the building of effective hadronic Hamiltonians in the context of effective field theories [22], as it implements in a certain sense the "quasiparticle" method of Weinberg [23]. In Weinberg's quasiparticle approach the bound states are redescribed by elementary particles and, in order not to change the physics of the problem, the potential is modified in such a way that it cannot produce these bound states any longer. In the Fock-Tani representation, as a result of the transformation of the quark Hamiltonian, the quark-quark interactions become "weaker", in the sense that they describe only quark-quark scattering processes and cannot produce the hadrons as bound states. The interesting feature of the Fock-Tani representation is that the change of the potential is the result of the unitary transformation that implements the mapping of composite hadrons into elementary hadrons, while in Weinberg's approach there is some freedom in how the potential is modified. Other appealing features of the Fock-Tani representation are: (a) it can be naturally extended to composites with any number of constituents, not only pairs or triplets of fermions, (b) systems of composite bosons and fermions, i.e. systems containing simultaneously mesons and baryons, can be naturally treated in a unified manner, and (c) it can be used with models where creation and annihilation of particles play an important role.

In the following section we review the basic ideas of the Fock-Tani representation. We introduce the unitary transformation for meson states of a quark-antiquark pair and derive 
the structure of the quark and antiquark operators in the new representation. Subsection $[\mathrm{A}$ contains the necessary extensions to the hadronic case of the original developments of Refs. [17,24, and incorporates the improvements and new aspects developed in Refs. [25,26]. In subsection [1B we introduce a model microscopic quark Hamiltonian, implement its Fock-Tani transformation, and discuss the physical interpretation of the different terms of the transformed Hamiltonian. In subsection 【IC, a commonly used quark Hamiltonian is then employed to compare the predictions of the Fock-Tani representation with other formalisms; in particular we compare results with Refs. [27,28], and discuss the implications of the post-prior symmetry [29] to an application of charmonium dissociation in matter [30]. In section [II] we construct the Fock-Tani transformation for the three-quark baryon systems; some results hereof were published previously in the form of a letter [31]. We perform the Fock-Tani transformation on the quark operators in subsection IIIA. The Hamiltonian that describes explicitly the baryon degrees of freedom in the new representation is obtained in subsection ПIIB. In subsection \#II we present an example of an effective nucleon-nucleon potential as derived from a microscopic spin-spin interaction and compare it to the one obtained by Barnes and collaborators [32] in the context of the Quark-Born diagram (QBD) method. Section IV contains a technical discussion about orthogonality corrections for the effective meson-meson and baryon-baryon Hamiltonian, and presents numerical calculations of their impact on the effective interactions. In section $\square$ we discuss the extension of the Fock-Tani representation to hadron Fock-space states that are more general than the quark-antiquark and the three quark states considered in previous sections. Such an extension is necessary for treating systems where creation and annihilation of particles plays an important role. Our conclusions and future perspectives are presented in section $\mathrm{VI}$.

\section{MAPPING OF MESONS}

This section reviews the formal aspects of the mapping procedure and implements it to quark-antiquark meson states. We start with the original formulation of Girardeau [15.17. and include new developments and improvements that have occurred since his original work. In section [II we implement the method for three-quark baryonic states. The more complicated Fock-space states are discussed in Section $\square$. 
The starting point of the Fock-Tani method is the definition of single composite bound states. We write a single-meson state in terms of a meson creation operator $M_{\alpha}^{\dagger}$ as

$$
\left|\alpha>=M_{\alpha}^{\dagger}\right| 0>
$$

where the meson creation operator $M_{\alpha}^{\dagger}$ is written in terms of constituent quark and antiquark creation operators $q^{\dagger}$ and $\bar{q}^{\dagger}$,

$$
M_{\alpha}^{\dagger}=\Phi_{\alpha}^{\mu \nu} q_{\mu}^{\dagger} \bar{q}_{\nu}^{\dagger}
$$

Here, $\Phi_{\alpha}^{\mu \nu}$ is the Fock-space amplitude of the meson, and $\mid 0>$ is the vacuum state, $q_{\mu} \mid 0>=$ $\bar{q}_{\nu} \mid 0>=0$. The index $\alpha$ identifies the quantum numbers of the meson, $\alpha=\{$ spatial, spin, isospin\}. The indices $\mu$ and $\nu$ denote the spatial, spin, flavor, and color quantum numbers of the constituent quarks. A summation over repeated indices is implied. It is convenient to work with orthonormalized amplitudes,

$$
<\alpha \mid \beta>=\Phi_{\alpha}^{* \mu \nu} \Phi_{\beta}^{\mu \nu}=\delta_{\alpha \beta}
$$

In Section [IB, a specific example of a quark model is discussed, and the short-hand notation for the labels of the states and operators will be spelled out in detail.

The quark and antiquark operators satisfy canonical anticommutation relations,

$$
\left\{q_{\mu}, q_{\nu}^{\dagger}\right\}=\left\{\bar{q}_{\mu}, \bar{q}_{\nu}^{\dagger}\right\}=\delta_{\mu \nu}, \quad\left\{q_{\mu}, q_{\nu}\right\}=\left\{\bar{q}_{\mu}, \bar{q}_{\nu}\right\}=\left\{q_{\mu}, \bar{q}_{\nu}\right\}=\left\{q_{\mu}, \bar{q}_{\nu}^{\dagger}\right\}=0
$$

Using these quark anticommutation relations, and the normalization condition of Eq. (3), it is easily shown that the meson operators satisfy the following noncanonical commutation relations

$$
\left[M_{\alpha}, M_{\beta}^{\dagger}\right]=\delta_{\alpha \beta}-\Delta_{\alpha \beta}, \quad\left[M_{\alpha}, M_{\beta}\right]=0,
$$

where

$$
\Delta_{\alpha \beta}=\Phi_{\alpha}^{* \mu \nu} \Phi_{\beta}^{\mu \sigma} \bar{q}_{\sigma}^{\dagger} \bar{q}_{\nu}+\Phi_{\alpha}^{* \mu \nu} \Phi_{\beta}^{\rho \nu} q_{\rho}^{\dagger} q_{\mu}
$$

In addition,

$$
\left[q_{\mu}, M_{\alpha}^{\dagger}\right]=\delta_{\mu \mu^{\prime}} \Phi_{\alpha}^{\mu^{\prime} \nu} \bar{q}_{\nu}^{\dagger}, \quad\left[\bar{q}_{\nu}, M_{\alpha}^{\dagger}\right]=-\delta_{\nu \nu^{\prime}} \Phi_{\alpha}^{\mu \nu^{\prime}} q_{\mu}^{\dagger}, \quad\left[q_{\mu}, M_{\alpha}\right]=\left[\bar{q}_{\nu}, M_{\alpha}\right]=0
$$


The presence of the operator $\Delta_{\alpha \beta}$ in Eq. (5) is due to the composite nature of the mesons. This term enormously complicates the mathematical description of processes that involve the hadron and quark degrees of freedom. The usual field theoretic techniques used in many-body problems, such as the Green's functions method, Wick's theorem, etc, apply to creation and annihilation operators that satisfy canonical relations. Similarly, the nonvanishing of the commutators $\left[q_{\mu}, M_{\alpha}^{\dagger}\right]$ and $\left[\bar{q}_{\nu}, M_{\alpha}^{\dagger}\right]$ is a manifestation of the lack of kinematic independence of the meson operator from the quark and antiquark operators. Therefore, the meson operators $M_{\alpha}$ and $M_{\alpha}^{\dagger}$ are not convenient dynamical variables to be used. Of course, the problem can be formulated in terms of the canonical constituent field operators only. But then there would be other difficulties, such as singularities in certain Green's functions due to the presence of bound states.

The bound state amplitude $\Phi_{\alpha}^{\mu \nu}$ is obtained from the microscopic quark-antiquark Hamiltonian. However, in implementing the Fock-Tani transformation, the explicit form of the microscopic Hamiltonian is not required; the method only requires knowledge of the form of the bound states in terms of the constituent operators. Therefore, the following discussion is completely general and does not depend on the details of the microscopic quark-antiquark interactions of the model. Once the transformation properties of the quark operators are obtained, the application of the transformation on a given microscopic model Hamiltonian gives rise to effective Hamiltonians that describe all possible processes involving quarks and hadrons.

The idea of the Fock-Tani method is to change representation, such that the field operators of composite particles are redescribed by field operators which satisfy canonical (anti)commutation relations. The complications of the composite nature of the hadrons will be shifted to the effective Hamiltonians. The main features of the Fock-Tani transformation are:

1. The transformation is performed by a unitary operator $U$, such that

$$
|\Omega>\longrightarrow| \Omega)=U^{-1} \mid \Omega>, \quad O \longrightarrow O_{\mathrm{FT}}=U^{-1} O U
$$

$\mid \Omega>$ is an arbitrary state vector and $O$ an arbitrary operator, both are expressed in terms of the constituent quark and antiquark operators $q, q^{\dagger}, \bar{q}, \bar{q}^{\dagger}$ of the original Fock 
representation. $(\Omega)$ and $O_{\mathrm{FT}}$ are the corresponding quantities in the new representation. Since $U$ is unitary, scalar products and matrix elements are preserved under the change of representation

$$
<\Omega|\Omega>=(\Omega \mid \Omega), \quad<\Omega| O \mid \Omega>=\left(\Omega\left|O_{\mathrm{FT}}\right| \Omega\right)
$$

Note that in the new representation, states are represented by rounded, instead of angular, bras and kets.

2. The transformation is defined such that a single-meson state $|\alpha\rangle$ is redescribed by an ("ideal") elementary-meson state by

$$
\left.\left.\left|\alpha>\longrightarrow U^{-1}\right| \alpha>\equiv \mid \alpha\right)=m_{\alpha}^{\dagger} \mid 0\right)
$$

where $m_{\alpha}^{\dagger}$ an ideal meson creation operator. The ideal meson operators $m_{\alpha}^{\dagger}$ and $m_{\alpha}$ satisfy, by definition, canonical commutation relations

$$
\left[m_{\alpha}, m_{\beta}^{\dagger}\right]=\delta_{\alpha \beta}, \quad\left[m_{\alpha}, m_{\beta}\right]=0
$$

The state $\mid 0)$ is the vacuum of both $q$ and $m$ degrees of freedom in the new representation. A precise definition of $\mid 0)$ is given later in the text. In addition, in the new representation the quark and antiquark operators $q^{\dagger}, q, \bar{q}^{\dagger}$ and $\bar{q}$ are kinematically independent of the $m_{\alpha}^{\dagger}$ and $m_{\alpha}$

$$
\left[q_{\mu}, m_{\alpha}\right]=\left[q_{\mu}, m_{\alpha}^{\dagger}\right]=\left[\bar{q}_{\mu}, m_{\alpha}\right]=\left[\bar{q}_{\mu}, m_{\alpha}^{\dagger}\right]=0
$$

3. A multi-meson state $\left|\alpha_{1}, \cdots, \alpha_{n}\right\rangle$, constructed from mutually non-overlapping and well-separated wave packets, is transformed into a multi-ideal-meson state $\left.\mid \alpha_{1}, \cdots, \alpha_{n}\right)$

$$
\left.\left.\left|\alpha_{1}, \cdots, \alpha_{n}>\rightarrow U^{-1}\right| \alpha_{1}, \cdots, \alpha_{n}>=\mid \alpha_{1}, \cdots, \alpha_{n}\right)=m_{\alpha_{1}}^{\dagger} \cdots m_{\alpha_{n}}^{\dagger} \mid 0\right) .
$$

This is particularly important for meson-meson scattering processes, where asymptotic states are non-overlapping. However, Eq. (13) is not true in the general case of multimeson states describing a dense system of mesons, where one expects considerable overlap among the mesons. 
4. Given a microscopic quark-antiquark Hamiltonian operator, the Fock-Tani transformed Hamiltonian can generally be written as

$$
H \rightarrow H_{\mathrm{FT}}=U^{-1} H U \equiv H_{\mathrm{FT}}^{(0)}+V_{\mathrm{FT}}
$$

$H_{\mathrm{FT}}^{(0)}$ is the "non-interacting" part; it contains the single-quark part of the original Hamiltonian, and a single-meson part which describes the free propagation of the composite mesons. $V_{\mathrm{FT}}$ is the interacting part, responsible for all possible interactions among the composites and the quarks. It describes only the "true" interaction processes, the binding effects of the quarks and antiquarks into mesons are contained in $H_{\mathrm{FT}}^{(0)}$.

The unitary operator $U$ that implements the transformation, Eq. (10), is constructed as follows: let the physical Fock space be denoted by $\mathcal{F}$. This is the space of states generated by all linear combinations of states constructed from the quark and antiquark operators $q^{\dagger}$ and $\bar{q}^{\dagger}$

$$
q_{\mu_{1}}^{\dagger} \cdots q_{\mu_{l}}^{\dagger} \bar{q}_{\nu_{1}}^{\dagger} \cdots \bar{q}_{\nu_{m}}^{\dagger} \mid 0>
$$

with $l$ and $m$ arbitrary. The quark and antiquark operators satisfy canonical anticommutation relations, Eq. (4), and $\mid 0>$ is the vacuum state defined as above.

Now, an independent Hilbert space $\mathcal{H}$, the "ideal hadron space" is defined, as the space of all linear combinations of states constructed from the ideal meson operators $\mathrm{m}^{\dagger}$,

$$
\left.m_{\alpha_{1}}^{\dagger} \cdots m_{\alpha_{n}}^{\dagger} \mid 0\right)_{\mathcal{H}}
$$

where $\mid 0)_{\mathcal{H}}$ is the vacuum of $\mathcal{H}$, that is, $\left.m_{\alpha} \mid 0\right)_{\mathcal{H}}=0$.

Next, the "ideal state space" $\mathcal{I}$ is defined as the graded direct product of the Fock space $\mathcal{F}$ and the ideal hadron space $\mathcal{H}: \mathcal{I}=\mathcal{F} \times \mathcal{H}$. By definition, the quark and ideal meson operators are kinematically independent and therefore satisfy Eq. (12) on $\mathcal{I}$. The commutation relations given in Eqs. (1) and (5), initially defined on $\mathcal{F}$, as well as those in Eq. (11), initially defined on $\mathcal{H}$, are also valid for $\mathcal{I}$.

The vacuum state of space $\mathcal{I}$ is the direct product of the vacua of $\mathcal{F}$ and $\mathcal{H}$. To simplify the notation, the symbol $\mid 0)$ is used to denote the product $\mid 0) \equiv|0>\times| 0)_{\mathcal{H}}$. Therefore, 
|0) is the vacuum of both the quark and ideal hadron degrees of freedom, $\left.\left.q_{\mu} \mid 0\right)=\bar{q}_{\mu} \mid 0\right)=$ $\left.m_{\alpha} \mid 0\right)=0$. The quark operators act on $\mid 0>$, and the ideal operators on $\left.\mid 0\right)_{\mathcal{H}}$.

The objective is to establish an one-to-one correspondence between the composite states in $\mathcal{F}$ and the ideal states of a subspace of $\mathcal{I}$. To that extent, we note that in $\mathcal{I}$ there is a subspace which is isomorphic to the original Fock space $\mathcal{F}$, namely, the space $\mathcal{I}_{0}$ consisting of those states $\mid \Omega>$ with no ideal mesons,

$$
m_{\alpha} \mid \Omega>=0
$$

This indicates that in $\mathcal{I}_{0}$ the ideal mesons are "redundant modes", in the language of the Bohm and Pines method [20]. As we shall discuss shortly, Eq. (17) is a constraint condition to ensure that there will be no double counting of degrees of freedom. This condition plays a role similar to the "negative particles" in the CPR of Ref. [3]. In the original formulation of the method [15] [17], the change of representation is performed by the operator

$$
U=\exp \left(-\frac{\pi}{2} F\right),
$$

where the generator of the transformation $F$ is given by

$$
F=m_{\alpha}^{\dagger} M_{\alpha}-M_{\alpha}^{\dagger} m_{\alpha},
$$

with $M_{\alpha}^{\dagger}$ given by Eq. (2). As before, a summation over repeated indices is implied. We note that $U$ acts on $\mathcal{I}$ and cannot be defined on $\mathcal{F}$. However, it is defined on $\mathcal{I}_{0}$, which is isomorphic to $\mathcal{F}$. The image $\mathcal{F}_{\mathrm{FT}}=U^{-1} \mathcal{I}_{0}$ of $\mathcal{I}_{0}$ is the subspace of $\mathcal{I}$ which consists of all states $\mid \Omega)$ in the new representation, related to the states $\mid \Omega>$ of $\mathcal{I}_{0}$ by

$$
\mid \Omega)=U^{-1} \mid \Omega>.
$$

In the subspace $\mathcal{F}_{\mathrm{FT}}$, Eq. (17) is transformed to

$$
\left.U^{-1} m_{\alpha} U \mid \Omega\right)=0
$$

Therefore, any calculation in the original Fock space is equivalent to a calculation in the Fock-Tani space $\mathcal{F}_{\mathrm{FT}}$ when Eq. (21) is satisfied. It is not difficult to prove [24] that the transformation implemented by such an operator $U$ does indeed have the characteristics 1 ) to 4) as discussed above. 
The great advantage of working in $\mathcal{F}_{\mathrm{FT}}$ is that all creation and annihilation operators satisfy canonical commutation relations. The transformed operators $O_{\mathrm{FT}}=U^{-1} O U$ give rise, in general, to an infinite series. This series physically represents an expansion in the "degree of overlap" of the composites in the system. For the derivation of an effective twomeson interaction, only a few terms in the series are necessary. A potential complication is the constraint equation, Eq. (21), called the "subsidiary condition". For scattering problems, where one starts with the proper definition of the asymptotic states, Eq. (21) is trivially satisfied. It is also satisfied in the particularly important case of the many ideal mesons state $\left.m_{\alpha_{1}}^{\dagger} \cdots m_{\alpha_{n}}^{\dagger} \mid 0\right)$ [26].

In the next subsection we present the derivation of the transformed quark operators.

\section{A. Transformation of the quark operators}

The next step is to obtain the transformed operators in the new representation. The basic operators of the model, such as the Hamiltonian, electromagnetic currents, etc, are expressed in terms of the quark operators. Therefore, in order to obtain the basic operators of the model in the new representation, the transformed quark operators are needed

$$
q_{\mathrm{FT}}=U^{-1} q U, \quad \bar{q}_{\mathrm{FT}}=U^{-1} \bar{q} U .
$$

The evaluation of such expressions by direct multi-commutators is tremendously difficult, it involves the summation of infinite series and cannot in general be expressed in a closed form. However, Girardeau [17] suggests the use of an "equations of motion" technique, which consists of the following. For any operator $\mathcal{O}$, a "time-dependent" operator is defined as

$$
\mathcal{O}(t)=\exp (-t F) \mathcal{O} \exp (t F),
$$

where $t$ is a real "time" parameter. Differentiating the above equation with respect to $t$, an equation of motion for $\mathcal{O}(t)$ is obtained,

$$
\frac{d \mathcal{O}(t)}{d t}=[\mathcal{O}(t), F],
$$

with the "initial condition" 


$$
\mathcal{O}(0)=0
$$

Therefore, the transformed operators are obtained from the solution of Eqs. (24) and (25) by setting $t=-\pi / 2$ at the end

$$
\mathcal{O}_{\mathrm{FT}}=U^{-1} \mathcal{O} U=\mathcal{O}(-\pi / 2)
$$

The equations of motion for the quark operators $q$ and $\bar{q}$ can be obtained by making use of Eq. (7), which leads to

$$
\begin{aligned}
& \frac{d q_{\mu}(t)}{d t}=\left[q_{\mu}(t), F\right]=-\delta_{\mu \mu_{1}} \Phi_{\alpha}^{\mu_{1} \nu} \bar{q}_{\nu}^{\dagger}(t) m_{\alpha}(t), \\
& \frac{d \bar{q}_{\nu}(t)}{d t}=\left[\bar{q}_{\nu}(t), F\right]=\delta_{\nu \nu_{1}} \Phi_{\alpha}^{\mu \nu_{1}} q_{\mu}^{\dagger}(t) m_{\alpha}(t) .
\end{aligned}
$$

Since these equations involve $m_{\alpha}(t)$, the equation of motion for $m_{\alpha}(t)$ is also needed

$$
\frac{d m_{\alpha}(t)}{d t}=\left[m_{\alpha}(t), F\right]=M_{\alpha}(t) .
$$

The system of equations is closed with

$$
\frac{d M_{\alpha}(t)}{d t}=\left[O_{\alpha}(t), F\right]=-\left[\delta_{\alpha \beta}-\Delta_{\alpha \beta}(t)\right] m_{\beta}(t) .
$$

Eqs. (27)-(30) (together with their hermitian conjugates) form a set of nonlinear ordinary differential equations. Obviously this set of equations is as complicated to solve as the evaluation of the multicommutators discussed above. However, these equations can be solved in a straightforward way by iteration. Starting from a "zero-order" approximation, where the overlap among the mesons is neglected, terms of the same "power" in the bound state amplitudes $\Phi_{\alpha}$ and $\Phi_{\alpha}^{*}$ are collected. For each operator the expansions are then written,

$$
q_{\mu}(t)=\sum_{i=1}^{\infty} q_{\mu}^{(i)}(t), \quad \bar{q}_{\mu}(t)=\sum_{i=1}^{\infty} \bar{q}_{\mu}^{(i)}(t), \quad m_{\alpha}(t)=\sum_{i=1}^{\infty} m_{\alpha}^{(i)}(t), \quad M_{\alpha}(t)=\sum_{i=1}^{\infty} M_{\alpha}^{(i)}(t),
$$

where the superscript $i$ denotes the $i$-th power of $\Phi_{\alpha}$ and $\Phi_{\alpha}^{*}$ in the series. In order to have a consistent power counting, the implicit $\Phi_{\alpha}$ and $\Phi_{\alpha}^{*}$ entering via Eq. (2) are not counted [17]. In order to derive an effective meson-meson interaction, the expansion up to the third order in the wave functions [17] is needed. Therefore, we will explicitly derive the transformed operators up to the third order. 
In the zeroth-order approximation, the effects of the meson structure are neglected. This amounts to neglect the terms $\Delta_{\alpha \beta}$ and $\Phi_{\alpha}^{\mu \nu}$ of Eqs. (27) , (28) and (30):

$$
\frac{d q_{\mu}^{(0)}(t)}{d t}=0, \quad \frac{d \bar{q}_{\nu}^{(0)}(t)}{d t}=0, \quad \frac{d M_{\alpha}^{(0)}(t)}{d t}=-m_{\alpha}^{(0)}(t), \quad \frac{d m_{\alpha}^{(0)}(t)}{d t}=M_{\alpha}^{(0)}(t) .
$$

Using the initial condition, Eq. (25), the zero-order solutions are found to be:

$$
\begin{aligned}
& q_{\mu}^{(0)}(t)=q_{\mu}, \quad \bar{q}_{\nu}^{(0)}(t)=\bar{q}_{\nu}, \\
& m_{\alpha}^{(0)}(t)=m_{\alpha} \cos t+M_{\alpha} \sin t, \quad M_{\alpha}^{(0)}(t)=M_{\alpha} \cos t-m_{\alpha} \sin t
\end{aligned}
$$

At this zeroth-order approximation, the transformed mesons behave like elementary bosons, and the transformation of $M_{\alpha}$ to $m_{\alpha}$ has the interpretation of a rotation of $-\pi / 2$ in the space $\mathcal{I}$.

The first-order equations are given by

$$
\begin{aligned}
& \frac{d q_{\mu}^{(1)}(t)}{d t}=-\delta_{\mu \mu_{1}} \Phi_{\alpha}^{\mu_{1} \nu_{1}} \bar{q}_{\nu_{1}}^{\dagger(0)}(t) m_{\alpha}^{(0)}(t), \quad \frac{d \bar{q}_{\nu}^{(1)}(t)}{d t}=\delta_{\nu \nu_{1}} \Phi_{\alpha}^{\mu_{1} \nu_{1}} q_{\mu_{1}}^{\dagger(0)}(t) m_{\alpha}^{(0)}(t), \\
& \frac{d M_{\alpha}^{(1)}(t)}{d t}=-m_{\alpha}^{(1)}(t), \quad \frac{d m_{\alpha}^{(1)}(t)}{d t}=M_{\alpha}^{(1)}(t) .
\end{aligned}
$$

Since the initial conditions were assigned to the zero-order terms, one obtains

$$
q_{\mu}^{(i)}(t=0)=\bar{q}_{\mu}^{(i)}(t=0)=m_{\alpha}^{(i)}(t=0)=M_{\alpha}^{(i)}(t=0)=0, \text { for } i \geq 1
$$

Therefore, the solutions to the first-order equations can be written as

$$
\begin{aligned}
& q_{\mu}^{(1)}(t)=-\delta_{\mu \mu_{1}} \Phi_{\alpha}^{\mu_{1} \nu_{1}} \bar{q}_{\nu_{1}}^{\dagger}\left[m_{\alpha} \sin t+M_{\alpha}(1-\cos t)\right], \\
& \bar{q}_{\nu}^{(1)}(t)=+\delta_{\nu \nu_{1}} \Phi_{\alpha}^{\mu_{1} \nu_{1}} q_{\mu_{1}}^{\dagger}\left[m_{\alpha} \sin t+M_{\alpha}(1-\cos t)\right], \\
& m_{\alpha}^{(1)}(t)=0, \quad M_{\alpha}^{(1)}(t)=0 .
\end{aligned}
$$

The iterative procedure can be continued to higher orders in a straightforward way. However, the solutions of second and higher orders will give rise to secular terms; i.e. terms which involve polynomials in $t$, in addition to trigonometric functions in $t$. Among other problems, the secular terms introduce the familiar post-prior discrepancies in the analysis of scattering and reactive processes. The origin of the secular terms is the asymmetry of the equations of motion for $m_{\alpha}(t)$ and $M_{\alpha}(t)$, Eqs. (29) and (30); the term proportional to 
$\Delta_{\alpha \beta}$ spoils the symmetry. The solution to the problem was recently found by Girardeau and Straton [25]. The solution consists in adding to $F$ a term depending on $\Delta_{\alpha \beta}$, such that the equations become symmetrical.

Here, a more systematic and elegant, although equivalent procedure of Lo and Girardeau [26] is followed. The generator of the transformation $F$ is generalized to

$$
F=m_{\alpha}^{\dagger} O_{\alpha}-O_{\alpha}^{\dagger} m_{\alpha}
$$

where the operator $O_{\alpha}$ is a function of the quark and antiquark operators, and are chosen such that

$$
\left[O_{\alpha}, O_{\beta}^{\dagger}\right]=\delta_{\alpha \beta}, \quad\left[O_{\alpha}, O_{\beta}\right]=\left[O_{\alpha}^{\dagger}, O_{\beta}^{\dagger}\right]=0 .
$$

The consequence of this is that the equations for $m_{\alpha}$ and $O_{\alpha}$ are manifestly symmetric,

$$
\frac{d m_{\alpha}(t)}{d t}=\left[m_{\alpha}(t), F\right]=O_{\alpha}(t), \quad \frac{d O_{\alpha}(t)}{d t}=\left[O_{\alpha}(t), F\right]=-m_{\alpha}(t),
$$

and their solutions involve only trigonometric functions of $t$,

$$
m_{\alpha}(t)=O_{\alpha} \sin t+m_{\alpha} \cos t, \quad O_{\alpha}(t)=O_{\alpha} \cos t-m_{\alpha} \sin t .
$$

The operators $O_{\alpha}$ are obtained order-by-order in a expansion in power of the bound state wave functions, as follows. In the zeroth-order, it is clear that

$$
O_{\alpha}^{(0)}=M_{\alpha}
$$

This certainly satisfies Eq. (40) to zeroth order, and reproduces the original results at the zeroth and first orders. Since there is no first order contribution to $O_{\alpha}$, because $\Delta_{\alpha \beta}$, which is the term to be canceled in the commutation relation, is already of the second order. Therefore,

$$
O_{\alpha}=M_{\alpha}+O_{\alpha}^{(2)}
$$

where $O_{\alpha}^{(2)}$ must be chosen such that

$$
\left[O_{\alpha}, O_{\beta}^{\dagger}\right]=\delta_{\alpha \beta}+\mathcal{O}\left(\Phi^{3}\right)
$$

The appropriate choice is easily checked to be: 


$$
O_{\alpha}^{(2)}=\frac{1}{2} \Delta_{\alpha \beta} M_{\beta}
$$

Following the same course, the appropriate third order operator $O_{\alpha}^{(3)}$ is discovered to be given by

$$
O_{\alpha}^{(3)}=-\frac{1}{2} M_{\beta}^{\dagger}\left[\Delta_{\beta \gamma}, M_{\alpha}\right] M_{\gamma}
$$

Note that a sum over repeated indices is implied in Eqs. (46) and (47).

Using the new generator F, Eq. (39), the second order equations for the quark and antiquark operators are obtained in a straightforward way. They are given by

$$
\begin{aligned}
\frac{d q_{\mu}^{(2)}(t)}{d t} & =-\delta_{\mu \mu_{1}}\left[\Phi_{\alpha}^{\mu_{1} \nu_{1}} \bar{q}_{\nu_{1}}^{\dagger(1)}(t) m_{\alpha}^{(0)}(t)+\frac{1}{2} \Phi_{\alpha}^{* \mu_{2} \nu_{1}} \Phi_{\beta}^{\mu_{1} \nu_{1}} m_{\alpha}^{\dagger(0)}(t) q_{\mu_{2}}^{(0)}(t) M_{\beta}^{(0)}(t)\right. \\
& \left.-\frac{1}{2} \Phi_{\alpha}^{* \mu_{2} \nu_{1}} \Phi_{\beta}^{\mu_{1} \nu_{1}} M_{\alpha}^{\dagger(0)}(t) q_{\mu_{2}}^{(0)}(t) m_{\beta}^{(0)}(t)\right] \\
\frac{d \bar{q}_{\nu}^{(2)}(t)}{d t} & =\delta_{\nu \nu_{1}}\left[\Phi_{\alpha}^{\mu_{1} \nu_{1}} q_{\mu_{1}}^{\dagger(0)}(t) m_{\alpha}^{(0)}(t)+\frac{1}{2} \Phi_{\alpha}^{* \mu_{1} \nu_{2}} \Phi_{\beta}^{\mu_{1} \nu_{1}} m_{\alpha}^{\dagger(0)}(t) M_{\beta}^{(0)}(t) \bar{q}_{\nu_{2}}^{(0)}(t)\right. \\
& \left.-\frac{1}{2} \Phi_{\alpha}^{* \mu_{1} \nu_{2}} \Phi_{\beta}^{\mu_{1} \nu_{1}} M_{\alpha}^{\dagger(0)}(t) m_{\beta}^{(0)}(t) \bar{q}_{\nu_{2}}^{(0)}(t)\right]
\end{aligned}
$$

The integration of these equations leads to the following expressions

$$
\begin{aligned}
q_{\mu}^{(2)}(t) & =\delta_{\mu \mu_{1}} \frac{1}{2} \Phi_{\alpha}^{* \mu_{2} \nu_{1}} \Phi_{\beta}^{\mu_{1} \nu_{1}}\left[m_{\alpha}^{\dagger} M_{\beta} \sin t \cos t-m_{\alpha}^{\dagger} m_{\beta} \sin ^{2} t-M_{\alpha}^{\dagger} M_{\beta}(1-\cos t)^{2}\right. \\
& \left.-M_{\alpha}^{\dagger} m_{\beta}(2-\cos t) \sin t\right] q_{\mu_{2}} \\
\bar{q}_{\nu}^{(2)}(t) & =\delta_{\nu \nu_{1}} \frac{1}{2} \Phi_{\alpha}^{* \mu_{1} \nu_{2}} \Phi_{\beta}^{\mu_{1} \nu_{1}}\left[m_{\alpha}^{\dagger} M_{\beta} \sin t \cos t-m_{\alpha}^{\dagger} m_{\beta} \sin ^{2} t-M_{\alpha}^{\dagger} M_{\beta}(1-\cos t)^{2}\right. \\
& \left.-M_{\alpha}^{\dagger} m_{\beta}(2-\cos t) \sin t\right] \bar{q}_{\nu_{2}} .
\end{aligned}
$$

In the same way, one can derive the third order equations

$$
\begin{aligned}
\frac{d q_{\mu}^{(3)}(t)}{d t} & =-\delta_{\mu \mu_{1}} \frac{1}{2} \Phi_{\alpha}^{\mu_{1} \nu_{1}}\left\{2\left[\bar{q}_{\nu_{1}}^{\dagger(2)}(t) m_{\alpha}^{(0)}(t)+\bar{q}_{\nu_{1}}^{\dagger(0)}(t) m_{\alpha}^{(2)}(t)\right]-\bar{q}_{\nu_{1}}^{\dagger(0)}(t) \Delta_{\alpha \beta}(t) m_{\beta}^{(0)}(t)\right. \\
& +\Phi_{\beta}^{* \mu_{2} \nu_{1}}\left[m_{\beta}^{\dagger(0)}(t) q_{\mu_{2}}^{(1)}(t) M_{\alpha}^{(0)}(t)-M_{\beta}^{\dagger(0)}(t) q_{\mu_{2}}^{(1)}(t) m_{\alpha}^{(0)}(t)\right] \\
& \left.+\Phi_{\beta}^{* \rho \nu_{1}} \Phi_{\gamma}^{\rho \sigma} M_{\beta}^{\dagger(0)}(t) \bar{q}_{\sigma}^{\dagger(0)}(t)\left[M_{\gamma}^{(0)}(t) m_{\alpha}^{(0)}(t)+M_{\alpha}^{(0)}(t) m_{\gamma}^{(0)}(t)\right]\right\}, \\
\frac{d \bar{q}_{\nu}^{(3)}(t)}{d t} & =\delta_{\nu \nu_{1}} \frac{1}{2} \Phi_{\alpha}^{\mu_{1} \nu_{1}}\left\{2\left[q_{\mu_{1}}^{\dagger(0)}(t) m_{\alpha}^{(2)}(t)+q_{\mu_{1}}^{\dagger(2)}(t) m_{\alpha}^{(0)}(t)\right]+q_{\mu_{1}}^{\dagger(0)}(t) \Delta_{\alpha \beta}(t) m_{\beta}^{(0)}(t)\right. \\
& +\Phi_{\beta}^{* \mu_{1} \sigma}\left[m_{\beta}^{\dagger(0)}(t) \bar{q}_{\sigma}^{(1)}(t) M_{\alpha}^{(0)}(t)-M_{\beta}^{\dagger(0)}(t) \bar{q}_{\sigma}^{(1)}(t) m_{\alpha}^{(0)}(t)\right] \\
& \left.-\Phi_{\beta}^{* \mu_{1} \sigma} \Phi_{\gamma}^{\rho \sigma} M_{\beta}^{\dagger(0)}(t) q_{\rho}^{\dagger(0)}(t)\left[M_{\alpha}^{(0)}(t) m_{\gamma}^{(0)}(t)+M_{\gamma}^{(0)}(t) m_{\alpha}^{(0)}(t)\right]\right\} .
\end{aligned}
$$


In order to integrate these equations $m_{\alpha}^{(2)}(t)$ is needed, which can be obtained from Eqs. (42) and (46)

$$
m_{\alpha}^{(2)}(t)=O_{\alpha}^{(2)} \sin t+m_{\alpha} \cos t=\frac{1}{2} \Delta_{\alpha \beta} M_{\beta} \sin t+m_{\alpha} \cos t .
$$

Using this in Eqs. (51) and (52) above, and integrating, the third order quark and antiquark operators are obtained

$$
\begin{aligned}
q_{\mu}^{(3)}(t) & =\delta_{\mu \mu_{1}} \frac{1}{2} \Phi_{\alpha}^{\mu_{1} \nu_{1}}\left\{\Phi _ { \beta } ^ { * \rho \nu _ { 1 } } \Phi _ { \gamma } ^ { \rho \sigma } \overline { q } _ { \sigma } ^ { \dagger } \left[m_{\beta}^{\dagger} m_{\alpha} m_{\gamma} \sin ^{3} t+M_{\beta}^{\dagger} M_{\alpha} m_{\gamma} \sin t \cos ^{2} t\right.\right. \\
& +M_{\beta}^{\dagger} m_{\alpha} M_{\gamma}\left(2-\cos t-\sin ^{2} t\right) \sin t-\left(M_{\beta}^{\dagger} m_{\alpha} m_{\gamma}+m_{\beta}^{\dagger} M_{\alpha} m_{\gamma}\right) \sin ^{2} t \cos t \\
& +m_{\beta}^{\dagger} m_{\alpha} M_{\gamma}(1-\cos t) \sin ^{2} t+M_{\beta}^{\dagger} M_{\alpha} M_{\gamma}\left(1+\cos ^{2} t\right)(1-\cos t) \\
& \left.\left.+m_{\beta}^{\dagger} M_{\alpha} M_{\gamma}(\cos t-1) \cos t \sin t\right]+\bar{q}_{\nu_{1}}^{\dagger} \Delta_{\alpha \beta}\left[2 M_{\beta}(\cos t-1)-m_{\beta} \sin t\right]\right\} \\
\bar{q}_{\nu}^{(3)}(t) & =-\delta_{\nu \nu_{1}} \frac{1}{2} \Phi_{\alpha}^{\mu_{1} \nu_{1}}\left\{\Phi _ { \beta } ^ { * \mu _ { 1 } \sigma } \Phi _ { \gamma } ^ { \rho _ { 1 } \sigma } q _ { \rho _ { 1 } } ^ { \dagger } \left[m_{\beta}^{\dagger} m_{\alpha} m_{\gamma} \sin ^{3} t+M_{\beta}^{\dagger} M_{\alpha} m_{\gamma} \sin t \cos ^{2} t\right.\right. \\
& +M_{\beta}^{\dagger} m_{\alpha} M_{\gamma}\left(2-\cos t-\sin ^{2} t\right) \sin t-\left(M_{\beta}^{\dagger} m_{\alpha} m_{\gamma}+m_{\alpha}^{\dagger} M_{\beta} m_{\gamma}\right) \sin ^{2} t \cos t \\
& +m_{\beta}^{\dagger} m_{\alpha} M_{\gamma}(1-\cos t) \sin ^{2} t+M_{\beta}^{\dagger} M_{\alpha} M_{\gamma}\left(1+\cos ^{2} t\right)(1-\cos t) \\
& \left.\left.+m_{\beta}^{\dagger} M_{\alpha} M_{\gamma}(\cos t-1) \cos t \sin t\right]+q_{\mu_{1}}^{\dagger} \Delta_{\alpha \beta}\left[2 M_{\beta}(1-\cos t)+m_{\beta} \sin t\right]\right\} .
\end{aligned}
$$

This completes the derivation of the transformed operators up to the third order. The formulae above provide the starting point for the construction of effective Hamiltonians for a variety of processes involving mesons and quarks, such as an effective meson-meson Hamiltonian. There are higher-order terms that provide orthogonality corrections to the lowest-order ones. These are rather trivial to obtain and so the discussion of this material is defered to section $\mathbb{\square}$.

\section{B. Effective Meson Hamiltonian}

The Fock-Tani Hamiltonian $H_{\mathrm{FT}}$ is obtained from the microscopic quark-antiquark Hamiltonian by the application of the unitary operator $U$, as indicated in Eq. (14). Therefore, the structure of the microscopic Hamiltonian must be specified. We consider a microscopic Hamiltonian of the general form

$$
\begin{aligned}
H & =T(\mu) q_{\mu}^{\dagger} q_{\mu}+T(\nu) \bar{q}_{\nu}^{\dagger} \bar{q}_{\nu} \\
& +V_{q \bar{q}}(\mu \nu ; \sigma \rho) q_{\mu}^{\dagger} \bar{q}_{\nu}^{\dagger} \bar{q}_{\rho} q_{\sigma}+\frac{1}{2} V_{q q}(\mu \nu ; \sigma \rho) q_{\mu}^{\dagger} q_{\nu}^{\dagger} q_{\rho} q_{\sigma}+\frac{1}{2} V_{\bar{q} \bar{q}}(\mu \nu ; \sigma \rho) \bar{q}_{\mu}^{\dagger} \bar{q}_{\nu}^{\dagger} \bar{q}_{\rho} \bar{q}_{\sigma} .
\end{aligned}
$$


The convention of a summation over repeated indices is again assumed. We note that a great variety of quark-model Hamiltonians used in the literature can be written in such a form. However, at this point of the discussion we have not included in Eq. (55) terms such as pair-creation, which are of the form $\bar{q}^{\dagger} q^{\dagger} q^{\dagger} q$. It will become clear from the discussion below that such terms can be handled with no additional problem. We also defer to section $\square$ the discussion of the more complicated structures of the Fock-space decomposition of meson states.

The transformation is implemented by transforming each quark and antiquark operator in Eq. (55). In free space, the single-meson Fock-space amplitudes $\Phi_{\alpha}^{\mu \nu}$ of Eq. (2) satisfy the following equation

$$
H\left(\mu \nu ; \mu^{\prime} \nu^{\prime}\right) \Phi_{\alpha}^{\mu^{\prime} \nu^{\prime}}=\epsilon_{[\alpha]} \Phi_{[\alpha]}^{\mu \nu},
$$

where $H\left(\mu \nu ; \mu^{\prime} \nu^{\prime}\right)$ is the Hamiltonian matrix

$$
H\left(\mu \nu ; \mu^{\prime} \nu^{\prime}\right)=\delta_{\mu\left[\mu^{\prime}\right]} \delta_{\nu\left[\nu^{\prime}\right]}\left[T\left(\left[\mu^{\prime}\right]\right)+T\left(\left[\nu^{\prime}\right]\right)\right]+V_{q \bar{q}}\left(\mu \nu ; \mu^{\prime} \nu^{\prime}\right)
$$

and $\epsilon_{[\alpha]}$ is the total energy of the meson (center-of-mass energy plus internal energy). We use the convention that there is no sum over repeated indices inside square brackets. We note that in order to implement the unitary transformation, there is no fundamental reason to use Fock-space amplitudes $\Phi_{\alpha}^{\mu \nu}$ that satisfy the free-space equation, Eq. (56) above. However, as we shall discuss shortly, such a choice facilitates many formal manipulations of the equations.

The transformed Hamiltonian $H_{\mathrm{FT}}=U^{-1} H U$ contains the same information as the original microscopic Hamiltonian, and as such describes all possible processes involving mesons and quarks. Such processes include two-body quark-quark, meson-quark, and mesonmeson interactions, as well as processes that involve more than two particles (quarks and mesons). As we already discussed previously, the unitary transformation can be evaluated, in general, only to a finite order in the meson Fock-space amplitude. This implies that only a limited class of processes are contained in the transformed Hamiltonian, evaluated up to a certain order. Up to the order that the quark operators are evaluated in this paper, it is possible to obtain an effective Hamiltonian that describes few-particle interactions. In the following, the discussion is specialized to the two-body processes, which are the quark-quark, meson-quark, and meson-meson processes. The derivation of the transformed 
Hamiltonian follows the same path as in Ref. [17] [26] for the case of atomic physics, where the bound states are of the same structure as our meson states. We have therefore skiped some intermediate steps, and refer the reader to noted references for details. In section [II baryons are considered, and since the method has not been implemented before for threeparticle bound states, the transformation of the Hamiltonian is presented with greater detail than in the present section.

The structure of the transformed Hamiltonian, $H_{\mathrm{FT}}=U^{-1} H U$ is of the following form

$$
H_{\mathrm{FT}}=H_{q}+H_{m}+H_{m q}
$$

where the first term involves only quark operators, the second one involves only ideal meson operators, and $H_{m q}$ involves quark and meson operators. A similar separation of the Hamiltonian is also explicitly obtained in the approach of Ref. [3] using the CPR. In Eq. (58], the quark Hamiltonian $H_{q}$ has an identical structure to the microscopic quark Hamiltonian, Eq. (55), except that the term corresponding to the quark-antiquark interaction is modified such that the new interaction does not produce quark-antiquark bound states. The new quark-antiquark interaction becomes modified as

$$
\begin{aligned}
V_{q \bar{q}} & =\left[V_{q \bar{q}}(\mu \nu ; \sigma \rho)-\Delta\left(\mu \nu ; \mu^{\prime} \nu^{\prime}\right) H\left(\mu^{\prime} \nu^{\prime} ; \sigma \rho\right)-H\left(\mu \nu ; \sigma^{\prime} \rho^{\prime}\right) \Delta\left(\sigma^{\prime} \rho^{\prime} ; \sigma \rho\right)\right. \\
& \left.+\Delta\left(\mu \nu ; \mu^{\prime} \nu^{\prime}\right) H\left(\mu^{\prime} \nu^{\prime} ; \sigma^{\prime} \rho^{\prime}\right) \Delta\left(\sigma^{\prime} \rho^{\prime} ; \sigma \rho\right)\right] q_{\mu}^{\dagger} \bar{q}_{\nu}^{\dagger} \bar{q}_{\rho} q_{\sigma}
\end{aligned}
$$

where $\Delta\left(\mu \nu ; \mu^{\prime} \nu^{\prime}\right)$ is the "bound state kernel"

$$
\Delta\left(\mu \nu ; \mu^{\prime} \nu^{\prime}\right)=\sum_{\alpha} \Phi_{\alpha}^{\mu \nu} \Phi_{\alpha}^{* \mu^{\prime} \nu^{\prime}}
$$

For the sake of clarity, here, and in a few subsequent formulas, we explicitly write the sum over repeated quantum numbers of mesons. One property of the bound state kernel we repeatedly make use of is

$$
\Delta\left(\mu \nu ; \mu^{\prime} \nu^{\prime}\right) \Phi_{\alpha}^{\mu^{\prime} \nu^{\prime}}=\Phi_{\alpha}^{\mu \nu}
$$

which follows from the orthonormalization of the $\Phi^{\prime}$ 's, Eq. (3). In the case that the $\Phi_{\alpha}^{\mu \nu}$ 's are stationary states of the microscopic Hamiltonian, i.e. they are solutions of Eq. (56), the quark-antiquark interaction term is modified as 


$$
V_{q \bar{q}}(\mu \nu ; \sigma \rho) \longrightarrow V_{q \bar{q}}(\mu \nu ; \sigma \rho)-\sum_{\alpha} \epsilon_{\alpha} \Phi_{\alpha}^{* \mu \nu} \Phi_{\alpha}^{\sigma \rho}
$$

where $\epsilon_{\alpha}$ is the meson total energy [see Eq. (56)]. It is not difficult to show that the spectrum of the modified quark Hamiltonian, $H_{q}$, is positive semi-definite and hence has no bound states [17]. This feature is exactly the same as in Weinberg's quasiparticle method [23]. In Weinberg's approach, the bound states are redescribed by elementary or ideal particles, and in order not to change the physics of the problem, the potential is modified in such a way that it cannot produce these bound states anymore. In the present formalism, this feature happens automatically.

The term involving only ideal meson operators has the general form

$$
H_{m}=T_{m}+V_{m m}
$$

where $T_{m}$ is the single-meson term,

$$
T_{m}=\sum_{\alpha \beta} \Phi_{\alpha}^{* \mu \nu} H\left(\mu \nu ; \mu^{\prime} \nu^{\prime}\right) \Phi_{\beta}^{\mu^{\prime} \nu^{\prime}} m_{\alpha}^{\dagger} m_{\beta}
$$

and $V_{m m}$ is the effective meson-meson interaction

$$
V_{m m}=\frac{1}{2} \sum_{\alpha \beta \gamma \delta} V_{m m}(\alpha \beta ; \gamma \delta) m_{\alpha}^{\dagger} m_{\beta}^{\dagger} m_{\delta} m_{\gamma}
$$

For later convenience, we have divided the potential into direct (dir), exchange (exc), and intra-exchange (int) parts as

$$
V_{m m}(\alpha \beta ; \gamma \delta)=V_{m m}^{d i r}(\alpha \beta ; \gamma \delta)+V_{m m}^{e x c}(\alpha \beta ; \gamma \delta)+V_{m m}^{i n t}(\alpha \beta ; \gamma \delta)
$$

where each of these is given by

$$
\begin{gathered}
V_{m m}^{d i r}(\alpha \beta ; \gamma \delta)=2 \Phi_{\alpha}^{* \mu \sigma} \Phi_{\beta}^{* \rho \nu} V_{q \bar{q}}\left(\mu \nu ; \mu^{\prime} \nu^{\prime}\right) \Phi_{\delta}^{\rho \nu^{\prime}} \Phi_{\gamma}^{\mu^{\prime} \sigma}+\Phi_{\alpha}^{* \mu \sigma} \Phi_{\beta}^{* \rho \nu} V_{q q}\left(\mu \rho ; \mu^{\prime} \rho^{\prime}\right) \Phi_{\delta}^{\rho^{\prime} \nu} \Phi_{\gamma}^{\mu^{\prime} \sigma} \\
+\Phi_{\alpha}^{* \mu \sigma} \Phi_{\beta}^{* \rho \nu} V_{\bar{q} \bar{q}}\left(\sigma \nu ; \sigma^{\prime} \nu^{\prime}\right) \Phi_{\delta}^{\rho \nu^{\prime}} \Phi_{\gamma}^{\mu \sigma^{\prime}} \\
V_{m m}^{e x c}(\alpha \beta ; \gamma \delta)=-\frac{1}{2}\left[\Phi_{\alpha}^{* \mu \nu} \Phi_{\beta}^{* \rho \sigma} V_{q \bar{q}}\left(\mu \nu ; \mu^{\prime} \nu^{\prime}\right) \Phi_{\delta}^{\mu^{\prime} \sigma} \Phi_{\gamma}^{\rho \nu^{\prime}}+\Phi_{\alpha}^{* \rho \sigma} \Phi_{\beta}^{* \mu \nu} V_{q \bar{q}}\left(\mu \nu ; \mu^{\prime} \nu^{\prime}\right) \Phi_{\delta}^{\rho \nu^{\prime}} \Phi_{\gamma}^{\mu^{\prime} \sigma}\right. \\
+\Phi_{\alpha}^{* \mu \sigma} \Phi_{\beta}^{* \rho \nu} V_{q \bar{q}}\left(\mu \nu ; \mu^{\prime} \nu^{\prime}\right) \Phi_{\delta}^{\mu^{\prime} \nu^{\prime}} \Phi_{\gamma}^{\rho \sigma}+\Phi_{\alpha}^{* \rho \nu} \Phi_{\beta}^{* \mu \sigma} V_{q \bar{q}}\left(\mu \nu ; \mu^{\prime} \nu^{\prime}\right) \Phi_{\delta}^{\rho \sigma} \Phi_{\gamma}^{\mu^{\prime} \nu^{\prime}} \\
\left.+2 \Phi_{\alpha}^{* \mu \sigma} \Phi_{\beta}^{* \rho \nu} V_{q q}\left(\mu \rho ; \mu^{\prime} \rho^{\prime}\right) \Phi_{\delta}^{\mu^{\prime} \nu} \Phi_{\gamma}^{\rho^{\prime} \sigma}+2 \Phi_{\alpha}^{* \mu \sigma} \Phi_{\beta}^{* \rho \nu} V_{\bar{q} \bar{q}}\left(\sigma \nu ; \sigma^{\prime} \nu^{\prime}\right) \Phi_{\delta}^{\mu \nu^{\prime}} \Phi_{\gamma}^{\rho \sigma^{\prime}}\right]
\end{gathered}
$$




$$
\begin{aligned}
V_{m m}^{i n t}(\alpha \beta ; \gamma \delta) & =-\frac{1}{2}\left[\Phi_{\alpha}^{* \mu \nu} \Phi_{\beta}^{* \rho \sigma} H\left(\mu \nu ; \mu^{\prime} \nu^{\prime}\right) \Phi_{\delta}^{\mu^{\prime} \sigma} \Phi_{\gamma}^{\rho \nu^{\prime}}+\Phi_{\alpha}^{* \rho \sigma} \Phi_{\beta}^{* \mu \nu} H\left(\mu \nu ; \mu^{\prime} \nu^{\prime}\right) \Phi_{\delta}^{\rho \nu^{\prime}} \Phi_{\gamma}^{\mu^{\prime} \sigma}\right. \\
& \left.+\Phi_{\alpha}^{* \mu \sigma} \Phi_{\beta}^{* \rho \nu} H\left(\mu \nu ; \mu^{\prime} \nu^{\prime}\right) \Phi_{\delta}^{\mu^{\prime} \nu^{\prime}} \Phi_{\gamma}^{\rho \sigma}+\Phi_{\alpha}^{* \rho \nu} \Phi_{\beta}^{* \mu \sigma} H\left(\mu \nu ; \mu^{\prime} \nu^{\prime}\right) \Phi_{\delta}^{\rho \sigma} \Phi_{\gamma}^{\mu^{\prime} \nu^{\prime}}\right]
\end{aligned}
$$

In general, the effective meson-meson potential is non-local, even when the microscopic interaction is a local one. The nonlocality is, of course, due to the extended structure of the mesons, and the size of the nonlocality is typically of the order of the size of the meson.

In section $\mathrm{IV}$ we will present a particularly interesting property of $V_{m m}^{\text {int }}$, namely, that it is canceled at the lowest order by orthogonality corrections. These orthogonality corrections have the same origin as the subtraction of the bound states from the microscopic quarkantiquark interaction, and in the present case they appear as powers of the bound state kernel $\Delta$, defined in Eq. (60). As a result of this cancelation, the effective meson-meson potential is then given by the first two terms in Eq. (66).

The term $H_{m q}$ describes a variety of processes involving mesons and quarks, such as quark-meson scattering, meson breakup into quarks, etc. One of these terms represents the two-meson breakup into two quarks and two antiquarks. The structure of such a term is

$$
H_{m m \rightarrow q \bar{q} q \bar{q}}=\frac{1}{2} V_{\alpha \beta}(\mu \nu ; \sigma \rho) q_{\mu}^{\dagger} \bar{q}_{\nu}^{\dagger} q_{\rho}^{\dagger} \bar{q}_{\sigma}^{\dagger} m_{\beta} m_{\alpha}
$$

where

$$
V_{\alpha \beta}(\mu \nu ; \sigma \rho)=V_{q q}\left(\mu \rho ; \mu^{\prime} \sigma^{\prime}\right) \Phi_{\alpha}^{\sigma^{\prime} \sigma} \Phi_{\beta}^{\mu^{\prime} \nu}+V_{\bar{q} \bar{q}}\left(\nu \sigma ; \nu^{\prime} \sigma^{\prime}\right) \Phi_{\alpha}^{\mu \sigma^{\prime}} \Phi_{\beta}^{\rho \nu^{\prime}}+2 V_{q \bar{q}}\left(\mu \sigma ; \mu^{\prime} \nu^{\prime}\right) \Phi_{\alpha}^{\mu^{\prime} \nu} \Phi_{\beta}^{\rho \nu^{\prime}} .
$$

When this term is iterated, together with its hermitian conjugate $H_{q \bar{q} q \bar{q} \rightarrow m m}=H_{m m \rightarrow q \bar{q} q \bar{q}}^{\dagger}$, which represents the recombination of two quarks and two antiquarks into two mesons, the following picture of a physical process occurs: (1) in the collision process the two mesons breakup into two quarks and two antiquarks under the action of $H_{m m \rightarrow q \bar{q} q \bar{q}}$, (2) then the quarks and antiquarks propagate freely, and (3) due to the confining forces they recombine into mesons under the action of $H_{q \bar{q} q \bar{q} \rightarrow m m}$. This mechanism is competitive with the one of the same second order Born approximation, where in the intermediate state the quarks and antiquarks remain bound into mesons. It would be interesting to investigate possible observable differences between these two mechanisms in a truly confining model, since this would allow to discriminate genuine quark effects from mesonic ones in the scattering process. 
Before proceeding to the next subsection, we notice that the Fock-space hadron amplitudes used to define the unitary operator $U$, in principle, do not need to be precisely the exact bound states of the original microscopic quark Hamiltonian. One might start with given "bare" amplitudes, with parameters to be determined at a later stage. The physical asymptotic states can always be obtained as "dressed" states that follow from the "bare" ones, using the effective Hamiltonians. That is, it is not necessary to obtain first the bound states of the microscopic Hamiltonian, and then perform the mapping. There might exist situations where the use of a set of "bare" amplitudes to generate the mapping is more convenient than using the complete set of exact bound states of the microscopic Hamiltonian.

In the next subsection, the application of the formalism for meson-meson scattering is illustrated through simple examples. We have specialized our applications in order to compare our results with existing calculations from the literature.

\section{Meson-meson scattering and post-prior symmetry}

In this subsection, the results are compared with other methods presented in the literature. We have compared our meson-meson results with the ones obtained recently in Refs. 27] 28] [30]. In order to do so, we use, as do the above references, a Fermi-Breit Hamiltonian, which includes the kinetic term, a spin-spin part of the one-gluon-exchange, and a gaussian potential for the confinement of the quarks and antiquarks. This choice is usually made for reasons of simplicity, as it allows one to perform almost all of the calculations analytically. We do not discuss the limitations and problems of such a model, since our aim is to compare results from the literature, and as such, we need to use the same model. In order to make the presentation self-contained, we begin with a collection of formulas relating the potential to scattering amplitudes and cross sections. We also present a brief discussion of the post-prior discrepancy, which is a problem that plagues composite-particle

formalisms. We then show explicitly, through a numerical example, the effect of the breaking of this symmetry and discuss how the present formalism solves the problem.

The exact two-meson scattering and reaction amplitudes are given by the $T$-matrix elements in the Fock-Tani representation as 


$$
T(\alpha \beta ; \gamma \delta)(z)=<\alpha \beta|T(z)| \gamma \delta>=\left(\alpha \beta\left|T_{F T}(z)\right| \gamma \delta\right)
$$

where the asymptotic final and initial meson states

$$
\begin{aligned}
& \left.\mid \alpha \beta)=U^{-1} M_{\alpha}^{\dagger} M_{\beta}^{\dagger}\left|0>=m_{\alpha}^{\dagger} m_{\beta}^{\dagger}\right| 0\right) \\
& \left.\mid \gamma \delta)=U^{-1} M_{\gamma}^{\dagger} M_{\delta}^{\dagger}\left|0>=m_{\gamma}^{\dagger} m_{\delta}^{\dagger}\right| 0\right)
\end{aligned}
$$

are eigenstates of the operator $T_{m}$ of Eq. (64). Note that for asymptotic scattering states, the unitary transformation is trivial, as discussed at the beginning of this section. The transition operator $T_{F T}(z)$ satisfies a Lippmann-Schwinger equation

$$
T_{F T}(z)=V_{F T}+V_{F T} G_{0}(z) T_{F T}(z)
$$

where $V_{F T}$ is the interacting part of the Fock-Tani Hamiltonian, and $G_{0}(z)$ is the noninteracting propagator

$$
G_{0}(z)=\left(z-H_{F T}^{(0)}\right)^{-1}
$$

Here, $H_{F T}^{(0)}$ corresponds to the single-particle part of $H_{F T}$

$$
H_{F T}^{(0)}=\sum_{\mu} T(\mu) q_{\mu}^{\dagger} q_{\mu}+\sum_{\nu} T(\nu) \bar{q}_{\nu}^{\dagger} \bar{q}_{\nu}+\sum_{\alpha \beta} \Phi_{\alpha}^{* \mu \nu} H\left(\mu \nu ; \mu^{\prime} \nu^{\prime}\right) \Phi_{\beta}^{\mu^{\prime} \nu^{\prime}} m_{\alpha}^{\dagger} m_{\beta}
$$

To first order, only the meson-meson part of the effective potential $V_{F T}$ contributes to the scattering matrix, since the asymptotic states contain only ideal mesons, and thus

$$
T\left(\alpha^{\prime} \beta^{\prime} ; \gamma^{\prime} \delta^{\prime}\right)(z)=\left(\alpha^{\prime} \beta^{\prime}\left|V_{m m}\right| \gamma^{\prime} \delta^{\prime}\right)
$$

The differential cross-section for the process $i \rightarrow f$ can be given in terms of the relativistic invariant matrix element, $\mathcal{M}_{f i}$, as

$$
\frac{d \sigma_{f i}(s, t, u)}{d t}=\frac{1}{64 \pi s} \frac{1}{P^{2}(s)}\left|\mathcal{M}_{f i}(s, t, u)\right|^{2},
$$

where $P(s)$ is the relative three-momentum of the initial mesons in the center-of-mass frame and $s, t, u$ are the Mandelstam variables. Due to translational invariance, the T-matrix element can be written as a momentum conservation delta-function, times a Born-order matrix element, $h_{f i}$ 


$$
T(\alpha \beta ; \gamma \delta)(z)=\delta^{(3)}\left(\boldsymbol{P}_{f}-\boldsymbol{P}_{i}\right) h_{f i},
$$

where $\boldsymbol{P}_{f}=\boldsymbol{p}_{\alpha}+\boldsymbol{p}_{\beta}$ and $\boldsymbol{P}_{i}=\boldsymbol{p}_{\gamma}+\boldsymbol{p}_{\delta}$ are the final and initial momenta of the two-meson system. The invariant matrix element is then given by

$$
\mathcal{M}_{f i}=\frac{1}{(2 \pi)^{3}} \prod_{n=1}^{4}\left[2(2 \pi)^{3} E_{n}\right]^{\frac{1}{2}} h_{f i}
$$

and the total cross-section is obtained by integrating Eq. (79) over t,

$$
\sigma_{f i}(s)=\int_{t_{-}}^{t_{+}} d t \frac{d \sigma_{f i}(s, t, u)}{d t}
$$

where $t_{-}$and $t_{+}$are the minimum and maximum transfer momenta .

Consider the rearrangement collision between composite particles $\gamma$ and $\delta$, resulting in particles $\alpha$ and $\beta, \gamma+\delta \rightarrow \alpha+\beta$. In this type of reaction, the constituents of the colliding systems can be redistributed during the process, and thus, the reaction may result in the same original states (elastic scattering) or in different states (inelastic scattering). The full Hamiltonian which describes the system is usually split into free and interaction parts as $H=H^{0}+V$. Because of the internal degrees of freedom, this decomposition is not unique [29]. One could, for instance, choose a decomposition in which $H^{0}$ is diagonal on the initial or final asymptotic states,

$$
H=H_{i}^{0}+V_{i}=H_{f}^{0}+V_{f} .
$$

Generally, $H_{i}^{0} \neq H_{f}^{0}$ and $V_{i} \neq V_{f}$, since the initial and final rearrangement channels can be different. Thus, the T-matrix element that describes the scattering can be written in either the prior form or in the post form, with the interaction respectively in either the initial channel or in the final channel

$$
T(\alpha \beta ; \gamma \delta)_{\text {prior }}=<\Psi_{\alpha \beta}^{-}\left|V_{i}\right| \phi_{\gamma \delta}>, \quad T(\alpha \beta ; \gamma \delta)_{\text {post }}=<\phi_{\alpha \beta}\left|V_{f}\right| \Psi_{\gamma \delta}^{+}>
$$

The initial and final free asymptotic states, $\phi_{\gamma \delta}$ and $\phi_{\alpha \beta}$, are eigenstates of the free Hamiltonians, $H_{i}^{0}$ and $H_{f}^{0}$. The outgoing and incoming exact scattering states, $\Psi_{\gamma \delta}^{+}$and $\Psi_{\alpha \beta}^{-}$, are eigenstates of the full Hamiltonian and satisfy Lippmann-Schwinger equations,

$$
\Psi_{\gamma \delta}^{+}=\phi_{\gamma \delta}+G_{0}^{+} V_{i} \Psi_{\gamma \delta}^{+}, \quad \Psi_{\alpha \beta}^{-}=\phi_{\alpha \beta}+G_{0}^{-} V_{f} \Psi_{\alpha \beta}^{-}
$$


where $G_{0}^{+}$and $G_{0}^{-}$are the retarded and advanced free-particle Green's functions given by

$$
G_{0}^{+}=\lim _{\epsilon \rightarrow 0^{+}}\left[E-H_{i}^{0}+i \epsilon\right]^{-1}, \quad G_{0}^{-}=\lim _{\epsilon \rightarrow 0^{+}}\left[E-H_{f}^{0}-i \epsilon\right]^{-1} .
$$

It can be proved that the prior and post forms are strictly equivalent on the energy shell 33. Thus, the exact T-matrix is symmetrical under the interchange of initial and final states:

$$
T(\alpha \beta ; \gamma \delta)=<\Psi_{\alpha \beta}^{-}\left|V_{i}\right| \phi_{\gamma \delta}>=<\phi_{\alpha \beta}\left|V_{f}\right| \Psi_{\gamma \delta}^{+}>.
$$

The first-Born approximation consists in replacing $\Psi_{\alpha \beta}^{-}$by $\phi_{\alpha \beta}$ and $\Psi_{\gamma \delta}^{+}$by $\phi_{\gamma \delta}$ in Eq. (84). Thus, the prior and post forms of the first-Born T-matrix elements are given by

$$
T_{B}(\alpha \beta ; \gamma \delta)_{\text {prior }}=<\phi_{\alpha \beta}\left|V_{i}\right| \phi_{\gamma \delta}>, \quad T_{B}(\alpha \beta ; \gamma \delta)_{\text {post }}=<\phi_{\alpha \beta}\left|V_{f}\right| \phi_{\gamma \delta}>.
$$

These two expressions are equal only if the free asymptotic states, $\phi_{\gamma \delta}$ and $\phi_{\alpha \beta}$, are exact eigenstates of their respective free Hamiltonians. When approximate wave functions are used for the bound states, one generally finds different values for the prior and post matrix elements. This difference is known as the "post-prior" discrepancy. It appears in many derivations of scattering amplitudes for reactions involving composite particles, since the exact bound state wave functions are usually not exactly known.

The lack of this symmetry will be shown not to be of importance for the "symmetric" initial and final states, as in the case of $\pi+\pi \rightarrow \pi+\pi$ [27] [28]; however, it is of importance for asymmetric cases like $J / \Psi+\pi \rightarrow D$-mesons [30]. Of course, one way to cure the problem, as done by Swanson in the first reference of Ref. [27], is to use the exact eigenstates of the free Hamiltonian. However, it might occur in a realistic situation that the problem is sufficiently complicated, and that an exact solution is unobtainable. Within the Fock-Tani formalism, a post-prior symmetrical effective Hamiltonian is automatically attained. As a result of this, a first-order T-matrix element that is free of post-prior discrepancy is obtained, even for approximate eigenstates of the "free" Hamiltonian.

Let us start specifying the quark-quark, antiquark-antiquark, and quark-antiquark terms in Eq. (55) that are used in Refs. [27] [28] [30]. Spelled out in full detail, these are given by

$$
\begin{aligned}
V_{q q}= & \frac{1}{2} \int \frac{d^{3} \boldsymbol{k}_{1} d^{3} \boldsymbol{k}_{2} d^{3} \boldsymbol{k}_{3} d^{3} \boldsymbol{k}_{4}}{(2 \pi)^{3}} \delta_{f_{1} f_{3}} \delta_{f_{2} f_{4}}\left(\frac{\lambda^{a}}{2}\right)^{c_{1} c_{3}}\left(\frac{\lambda^{a}}{2}\right)^{c_{2} c_{4}} \\
& \times U_{s_{1} s_{3} ; s_{2} s_{4}}\left(\boldsymbol{k}_{1}-\boldsymbol{k}_{3}\right) q_{s_{1} f_{1}}^{c_{1} \dagger}\left(\boldsymbol{k}_{1}\right) q_{s_{2} f_{2}}^{c_{2} \dagger}\left(\boldsymbol{k}_{2}\right) q_{s_{4} f_{4}}^{c_{4}}\left(\boldsymbol{k}_{4}\right) q_{s_{3} f_{3}}^{c_{3}}\left(\boldsymbol{k}_{3}\right),
\end{aligned}
$$




$$
\begin{aligned}
V_{\bar{q} \bar{q}}=\frac{1}{2} & \int \frac{d^{3} \boldsymbol{k}_{1} d^{3} \boldsymbol{k}_{2} d^{3} \boldsymbol{k}_{3} d^{3} \boldsymbol{k}_{4}}{(2 \pi)^{3}} \delta_{f_{1} f_{3}} \delta_{f_{2} f_{4}}\left(\frac{\lambda^{a T}}{2}\right)^{c_{1} c_{3}}\left(\frac{\lambda^{a T}}{2}\right)^{c_{2} c_{4}} \\
& \times U_{s_{1} s_{3} ; s_{2} s_{4}}\left(\boldsymbol{k}_{1}-\boldsymbol{k}_{3}\right) \bar{q}_{s_{1} f_{1}}^{c_{1} \dagger}\left(\boldsymbol{k}_{1}\right) \bar{q}_{s_{2} f_{2}}^{c_{2} \dagger}\left(\boldsymbol{k}_{2}\right) \bar{q}_{s_{4} f_{4}}^{c_{4}}\left(\boldsymbol{k}_{4}\right) \bar{q}_{s_{3} f_{3}}^{c_{3}}\left(\boldsymbol{k}_{3}\right), \\
V_{q \bar{q}}=- & \int \frac{d^{3} \boldsymbol{k}_{1} d^{3} \boldsymbol{k}_{2} d^{3} \boldsymbol{k}_{3} d^{3} \boldsymbol{k}_{4}}{(2 \pi)^{3}} \delta_{f_{1} f_{3}} \delta_{f_{2} f_{4}}\left(\frac{\lambda^{a}}{2}\right)^{c_{1} c_{3}}\left(\frac{\lambda^{a T}}{2}\right)^{c_{2} c_{4}} \\
& \times U_{s_{1} s_{3} ; s_{2} s_{4}}\left(\boldsymbol{k}_{1}-\boldsymbol{k}_{3}\right) q_{s_{1} f_{1}}^{c_{1} \dagger}\left(\boldsymbol{k}_{1}\right) \bar{q}_{s_{2} f_{2}}^{c_{2} \dagger}\left(\boldsymbol{k}_{2}\right) \bar{q}_{s_{4} f_{4}}^{c_{4}}\left(\boldsymbol{k}_{4}\right) q_{s_{3} f_{3}}^{c_{3}}\left(\boldsymbol{k}_{3}\right),
\end{aligned}
$$

where $\lambda^{a} / 2$ for quark and $-\lambda^{a T} / 2$ (T means transpose) for antiquarks, are the $S U(3)$ color matrices. The potential functions are given by

$$
U_{s_{1} s_{3} ; s_{2} s_{4}}(\boldsymbol{q})=\delta^{(3)}\left(\boldsymbol{k}_{1}+\boldsymbol{k}_{2}-\boldsymbol{k}_{3}-\boldsymbol{k}_{4}\right)\left[U_{s_{1} s_{3} ; s_{2} s_{4}}^{c \cos }(\boldsymbol{q})+U_{s_{1} s_{3} ; s_{2} s_{4}}^{s s}(\boldsymbol{q})\right]
$$

with

$$
\begin{aligned}
& U_{s_{1} s_{3} ; s_{2} s_{4}}^{c o n f}(\boldsymbol{q})=\delta_{s_{1} s_{3}} \delta_{s_{2} s_{4}}\left[V_{0}\left(\frac{4 \pi}{\chi}\right)^{\frac{3}{2}} e^{-\boldsymbol{q}^{2} / \chi}+C\right] \\
& U_{s_{1} s_{3} ; s_{2} s_{4}}^{s_{1}}(\boldsymbol{q})=-\frac{8 \pi \alpha_{s}}{3 m_{1} m_{2}}\left(\frac{\sigma^{i}}{2}\right)_{s_{1} s_{3}}\left(\frac{\sigma^{i}}{2}\right)_{s_{2} s_{4}},
\end{aligned}
$$

where $m_{1}$ and $m_{2}$ are the constituent quark and antiquark masses, $\sigma^{i}(i=1,2,3)$ are the Pauli spin matrices, $\alpha_{s}$ is the QCD structure constant, $V_{0}$ and $\chi$ are the parameters of the gaussian potential, and $C$ is a constant to adjust the lower part of the spectrum.

The explicit form of the creation operator for a composite meson is

$$
M_{C S F}^{\dagger}(\boldsymbol{p})=\sum_{c s f} \mathcal{C}_{C}^{c_{1} c_{2}} \chi_{S}^{s_{1} s_{2}} \mathcal{F}_{F}^{f_{1} f_{2}} \int d \boldsymbol{k}_{1} d \boldsymbol{k}_{2} \Phi_{\boldsymbol{p}}\left(\boldsymbol{k}_{1}, \boldsymbol{k}_{2}\right) q_{c_{1} s_{1} f_{1}}^{\dagger}\left(\boldsymbol{k}_{1}\right) \bar{q}_{c_{2} s_{2} f_{2}}^{\dagger}\left(\boldsymbol{k}_{2}\right)
$$

where $\mathcal{C}_{C}, \chi_{S}$, and $\mathcal{F}_{F}$ are respectively the color, spin and flavor Clebsch-Gordan coefficients, and $\Phi_{\boldsymbol{p}}$ is the spatial amplitude, an S-wave gaussian given by

$$
\Phi_{\boldsymbol{p}}^{\boldsymbol{k}_{1} \boldsymbol{k}_{2}}=\delta^{(3)}\left(\boldsymbol{p}-\boldsymbol{k}_{1}-\boldsymbol{k}_{2}\right)\left(\frac{b^{2}}{\pi}\right)^{\frac{3}{4}} e^{-b^{2} \overline{\boldsymbol{k}}^{2} / 2},
$$

where $\overline{\boldsymbol{k}}=\eta \boldsymbol{k}_{1}-(1-\eta) \boldsymbol{k}_{2}$, with $\eta=m_{2} /\left(m_{1}+m_{2}\right)$. The gaussian parameter $b$ is related to the r.m.s. radius of the meson by $\left\langle r^{2}\right\rangle=\sqrt{3 / 2} b$.

Let us now compare our results with the ones from Ref. [27], which uses the QBD method, and those from Ref. [28], which uses a Green's functions method. These references consider $I=2$ meson-meson scattering, and consider $m_{1}=m_{2}=m_{q}$. In the present formalism the effective meson-meson potential for this channel is given by 


$$
\begin{aligned}
V_{m m}= & \frac{1}{2} \int d^{3} \boldsymbol{P} d^{3} \boldsymbol{P}^{\prime} d^{3} \boldsymbol{p} d^{3} \boldsymbol{p}^{\prime} \delta^{(3)}\left(\boldsymbol{P}^{\prime}-\boldsymbol{P}\right)\left[V_{c o n f}\left(\boldsymbol{p}, \boldsymbol{p}^{\prime}\right)+V_{s s}\left(\boldsymbol{p}, \boldsymbol{p}^{\prime}\right)\right] \\
& \times m_{\lambda_{1}}^{\dagger}\left(\boldsymbol{P}^{\prime} / 2+\boldsymbol{p}^{\prime}\right) m_{\lambda_{2}}^{\dagger}\left(\boldsymbol{P}^{\prime} / 2-\boldsymbol{p}^{\prime}\right) m_{\lambda_{4}}(\boldsymbol{P} / 2-\boldsymbol{p}) m_{\lambda_{3}}(\boldsymbol{P} / 2+\boldsymbol{p}),
\end{aligned}
$$

where $\lambda_{i}(i=1, \cdots 4)$ are the meson isospin quantum numbers (in this example they correspond to the quantum numbers of $\pi^{+}$), and $V_{\text {conf }}$ and $V_{s s}$ are respectively the contributions from the confining and the spin-spin interactions, given by

$$
\begin{aligned}
& V_{\text {conf }}\left(\boldsymbol{p}, \boldsymbol{p}^{\prime}\right)=\frac{2 V_{0} b^{3}}{9}\left(\frac{z}{2 \pi}\right)^{\frac{3}{2}}\left\{\frac{8}{[z(4+3 x)]^{\frac{3}{2}}}\left[e^{-b^{2}\left(\boldsymbol{p}^{\prime 2}+y \boldsymbol{p}^{2}\right) / 4}+e^{-b^{2}\left(y \boldsymbol{p}^{\prime 2}+\boldsymbol{p}^{2}\right) / 4}\right]\right.
\end{aligned}
$$

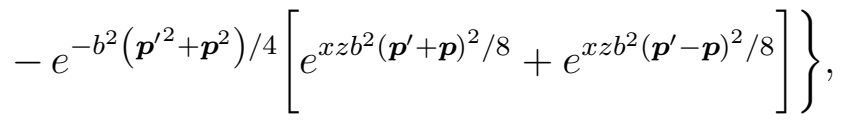

$$
\begin{aligned}
& V_{s s}\left(\boldsymbol{p}, \boldsymbol{p}^{\prime}\right)=\frac{\kappa_{s s}}{6}\left\{\frac{8}{3 \sqrt{3}}\left[e^{-b^{2}\left(\boldsymbol{p}^{\prime 2}+\boldsymbol{p}^{2} / 3\right) / 4}+e^{-b^{2}\left(\boldsymbol{p}^{2}+\boldsymbol{p}^{\prime 2} / 3\right) / 4}\right]\right. \\
& \left.+e^{-b^{2}\left(\boldsymbol{p}^{\prime}-\boldsymbol{p}\right)^{2} / 8}+e^{-b^{2}\left(\boldsymbol{p}^{\prime}+\boldsymbol{p}\right)^{2} / 8}\right\},
\end{aligned}
$$

where

$$
\kappa_{s s}=\frac{\alpha_{s}}{3 m_{q}^{2} \pi^{2}} \quad, \quad x=\frac{b^{2} \chi}{2} \quad, \quad y=\frac{(4+x)}{(4+3 x)} \quad, \quad z=\frac{1}{(1+x)} .
$$

Comparing our expressions with Eqs. (25) and (26) of Ref. [28], two differences are immediately noticed: (1) we have extra terms because in the meson-meson potential we have included the contributions coming from quark-quark and antiquark-antiquark interactions, whereas in Ref. 28] only the quark-antiquark was used, and (2) the different overall numerical coefficient is due to the fact that we have removed the color factors $\lambda^{a} \lambda^{a} / 4$ from the interactions. It is also noticeable from the above expressions that the effective meson-meson potential is symmetrical under the exchange of initial and final states $\left(\boldsymbol{p} \leftrightarrow \boldsymbol{p}^{\prime}\right)$.

Considering only the spin-spin term in Eq. (93), and writing the scattering amplitude $h_{f i}$ defined in Eq. (80) in terms of Mandelstam variables, the following Born-order on-shell matrix element is obtained:

$$
h_{f i}=\frac{\kappa_{s s}}{3}\left[\frac{16}{3 \sqrt{3}} e^{-b^{2}\left(s-4 M_{m e s}^{2}\right) / 12}+e^{b^{2} t / 8}+e^{b^{2} u / 8}\right],
$$

where

$$
M_{m e s}=2 m_{q}+\frac{3}{2 b^{2} m_{q}}-\frac{8 \alpha_{s}}{3 m_{q}^{2} b^{2} \sqrt{\pi}} .
$$


This result is identical to the one obtained in Ref. [27] with the QBD method.

Now we compare results for an "asymmetrical" reaction, namely, charmonium dissociation by inelastic scattering on pions 30

$$
J / \psi\left({ }^{3} 1 S_{1}\right)+\pi\left({ }^{1} 1 S_{0}\right) \rightarrow D(1 S)+\bar{D}(1 S) .
$$

The $J / \psi$ mesons are composites of a heavy quark and a heavy antiquark pair, denoted by $(Q \bar{Q})$, and the pions are composites of a light quark and a light antiquark, denoted by $(q \bar{q})$. The final mesons $D, \bar{D}$ are composites of a $(q \bar{Q})$ or a $(Q \bar{q})$ pair and can be either in the fundamental $D, \bar{D}\left({ }^{1} 1 S_{0}\right)$ or in the excited $D^{*}, \bar{D}^{*}\left({ }^{3} 1 S_{1}\right)$ states. By momentum conservation, the only allowed final meson states are the excited ones. The threshold for each process is given by the mass difference

$$
\Delta M=\left(M_{D}+M_{\bar{D}}\right)-\left(M_{J / \psi}+M_{\pi}\right)
$$

The cross-section for this process was calculated by Martins et al. [30 using the QBD. They employ the microscopic interaction given by Eqs. (93)-(95), but the confinement gaussian potential is not considered in the $q q$ and $\bar{q} \bar{q}$ channels, and the spin-spin term is neglected in all channels. In Ref. [30] the prior potential matrix elements are considered. In order to compare results, only the confinement contribution to the invariant matrix element is considered, and $V_{0}$ is multiplied by a numerical constant in order to compensate for the presence of Gell-Mann matrices in our Eq. (92).

In our formalism, the invariant matrix element can be written as

$$
\mathcal{M}_{f i}^{\text {conf }}=\frac{1}{2}\left[\left(\mathcal{M}_{q \bar{q} f}^{\text {conf }}\right)_{\text {prior }}+\left(\mathcal{M}_{q \bar{q}}^{\text {conf }}\right)_{\text {post }}\right]
$$

where the prior and post invariant matrix elements are obtained respectively from the prior and post effective potentials (note that because of the Gell-Mann matrices, the $V_{m m}^{d i r}$ part in Eq. (66) does not contribute)

$$
\begin{gathered}
V_{m m}(\alpha \beta ; \gamma \delta)_{\text {prior }}=-\left[\Phi_{\alpha}^{* \mu \nu} \Phi_{\beta}^{* \rho \sigma} V_{q \bar{q}}\left(\mu \nu ; \mu^{\prime} \nu^{\prime}\right) \Phi_{\delta}^{\mu^{\prime} \sigma} \Phi_{\gamma}^{\rho \nu^{\prime}}+\Phi_{\alpha}^{* \rho \sigma} \Phi_{\beta}^{* \mu \nu} V_{q \bar{q}}\left(\mu \nu ; \mu^{\prime} \nu^{\prime}\right) \Phi_{\delta}^{\rho \nu^{\prime}} \Phi_{\gamma}^{\mu^{\prime} \sigma}\right. \\
\left.+\Phi_{\alpha}^{* \mu \sigma} \Phi_{\beta}^{* \rho \nu} V_{q q}\left(\mu \rho ; \mu^{\prime} \rho^{\prime}\right) \Phi_{\delta}^{\mu^{\prime} \nu} \Phi_{\gamma}^{\rho^{\prime} \sigma}+\Phi_{\alpha}^{* \mu \sigma} \Phi_{\beta}^{* \rho \nu} V_{\bar{q} \bar{q}}\left(\sigma \nu ; \sigma^{\prime} \nu^{\prime}\right) \Phi_{\delta}^{\mu \nu^{\prime}} \Phi_{\gamma}^{\rho \sigma^{\prime}}\right] \\
V_{m m}(\alpha \beta ; \gamma \delta)_{p o s t}=-\left[\Phi_{\alpha}^{* \mu \sigma} \Phi_{\beta}^{* \rho \nu} V_{q \bar{q}}\left(\mu \nu ; \mu^{\prime} \nu^{\prime}\right) \Phi_{\delta}^{\mu^{\prime} \nu^{\prime}} \Phi_{\gamma}^{\rho \sigma}+\Phi_{\alpha}^{* \rho \nu} \Phi_{\beta}^{* \mu \sigma} V_{q \bar{q}}\left(\mu \nu ; \mu^{\prime} \nu^{\prime}\right) \Phi_{\delta}^{\rho \sigma} \Phi_{\gamma}^{\mu^{\prime} \nu^{\prime}}\right. \\
\left.+\Phi_{\alpha}^{* \mu \sigma} \Phi_{\beta}^{* \rho \nu} V_{q q}\left(\mu \rho ; \mu^{\prime} \rho^{\prime}\right) \Phi_{\delta}^{\mu^{\prime} \nu} \Phi_{\gamma}^{\rho^{\prime} \sigma}+\Phi_{\alpha}^{* \mu \sigma} \Phi_{\beta}^{* \rho \nu} V_{\bar{q} \bar{q}}\left(\sigma \nu ; \sigma^{\prime} \nu^{\prime}\right) \Phi_{\delta}^{\mu \nu^{\prime}} \Phi_{\gamma}^{\rho \sigma^{\prime}}\right]
\end{gathered}
$$


The term $\left(\mathcal{M}_{q \bar{q}}^{\text {conf }}\right)_{\text {prior }}$ is equal to the matrix element $a \bar{b}+\bar{a} b$ described in Appendix $\mathrm{C}$ of Ref. [30]. Using the notation of Ref. [30], one can write

$$
\left(\mathcal{M}_{q \bar{q}}^{\text {conf }}\right)_{\text {prior }}=\mathcal{M}_{f i}^{Q B D}=\mathcal{M}_{a \bar{b}}^{n p}+\mathcal{M}_{\bar{a} b}^{n p}
$$

That is, the prior form of our matrix element is one-half of $\mathcal{M}_{f i}^{Q B D}$ used in Ref. [30. The post form of the invariant amplitude is given by

$$
\left(\mathcal{M}_{q \bar{q}}^{\text {conf }}\right)_{\text {post }}=\mathcal{N} \frac{4}{9} \frac{V_{0}}{(2 \pi)^{3}}\left(\lambda_{Q Q} \lambda_{q q}\right)^{\frac{3}{4}}(32 \pi)^{\frac{3}{2}} x^{\frac{3}{2}} e^{-\alpha|\boldsymbol{P}|^{2}}\left(\frac{1}{\Lambda_{1}} e^{-\beta_{1}\left|\boldsymbol{P}^{\prime}\right|^{2}}+\frac{1}{\Lambda_{2}} e^{-\beta_{2}\left|\boldsymbol{P}^{\prime}\right|^{2}}\right)
$$

where $x=1 /(2 \chi), \alpha=\lambda_{Q q}$ and

$$
\begin{aligned}
& |\boldsymbol{P}|^{2}=\frac{1}{4 s}\left[\left(s-\left(M_{J / \psi}^{2}+M_{\pi}^{2}\right)^{2}-4 M_{J / \psi}^{2} M_{\pi}^{2}\right]\right. \\
& \left|\boldsymbol{P}^{\prime}\right|^{2}=\frac{1}{4 s}\left[\left(s-\left(M_{D}^{2}+M_{\bar{D}}^{2}\right)^{2}-4 M_{D}^{2} M_{\bar{D}}^{2}\right]\right. \\
& \mathcal{N}=\frac{(2 \pi)^{3}}{s}\left\{\left[s^{2}-\left(M_{J / \psi}^{2}-M_{\pi}^{2}\right)^{2}\right]\left[s^{2}-\left(M_{D}^{2}-M_{\bar{D}}^{2}\right)^{2}\right]\right\}^{\frac{1}{2}} \\
& \beta_{1}=\frac{2\left[2 \lambda_{Q q}\left(\lambda_{Q Q} \eta^{2}+\lambda_{q q}^{\prime}(1-\eta)^{2}\right)+\lambda_{q q}^{\prime} \lambda_{Q Q}\right]}{\lambda_{q q}^{\prime}+2 \lambda_{Q q}+\lambda_{Q Q}} \\
& \beta_{2}=\frac{2\left[2 \lambda_{Q q}\left(\lambda_{Q Q}^{\prime} \eta^{2}+\lambda_{q q}(1-\eta)^{2}\right)+\lambda_{q q} \lambda_{Q Q}^{\prime}\right]}{\lambda_{Q Q}^{\prime}+2 \lambda_{Q q}+\lambda_{q q}} \\
& \Lambda_{1}=\frac{\left(x+\lambda_{q q}\right)\left(\lambda_{q q}^{\prime}+2 \lambda_{Q q}+\lambda_{Q Q}\right)}{\lambda_{Q q}} \\
& \Lambda_{2}=\frac{\left(x+\lambda_{Q Q}\right)\left(\lambda_{Q Q}^{\prime}+2 \lambda_{Q q}+\lambda_{q q}\right)}{\lambda_{Q q}} .
\end{aligned}
$$

In these:

$$
\begin{aligned}
& \lambda_{Q q}=b_{D}^{2} / 4=b_{\bar{D}}^{2} / 4, \quad \lambda_{q q}=b_{\pi}^{2} / 4, \quad \lambda_{Q Q}=b_{J / \psi}^{2} / 4, \quad \eta=\frac{m_{Q}}{m_{q}+m_{Q}}, \\
& \lambda_{Q q}^{\prime}=\frac{x \lambda_{Q q}}{x+\lambda_{Q q}}, \quad \lambda_{q q}^{\prime}=\frac{x \lambda_{q q}}{x+\lambda_{q q}}, \quad \lambda_{Q Q}^{\prime}=\frac{x \lambda_{Q Q}}{x+\lambda_{Q Q}} .
\end{aligned}
$$

The cross-sections for the final channel are obtained from Eqs. (79) and (82). The total cross section for the reaction is a function of the center-of-mass energy and is obtained by summing over all possible final channels $\sigma_{t o t}(s)=\sum_{f=1}^{4} \sigma_{f i}(s)$. For numerical evaluations, the same parameter values as in Ref. [30] are used:

$$
\begin{aligned}
& m_{Q}=1.67 \mathrm{GeV}, m_{q}=0.33 \mathrm{GeV}, \\
& V_{0} \rightarrow \frac{3}{4} V_{0}=0.675 \mathrm{GeV}, \quad C=0.24 \mathrm{GeV}, \quad x=1.0 \mathrm{GeV}^{-2}, \\
& \lambda_{Q Q}=0.64 \mathrm{GeV}^{-2}, \quad \lambda_{q q}=2.4 \mathrm{GeV}^{-2}, \quad \lambda_{Q q}=1.7 \mathrm{GeV}^{-2} .
\end{aligned}
$$


In Fig. 1 the total cross sections for the $q \bar{q}$ channel is shown as a function of the relative kinetic energy of the $J / \psi$ and the $\pi$ in the center-of-mass system, $E$. The dashed line refers to Ref. [30], and the solid line is obtained with our post-prior symmetrical interaction.

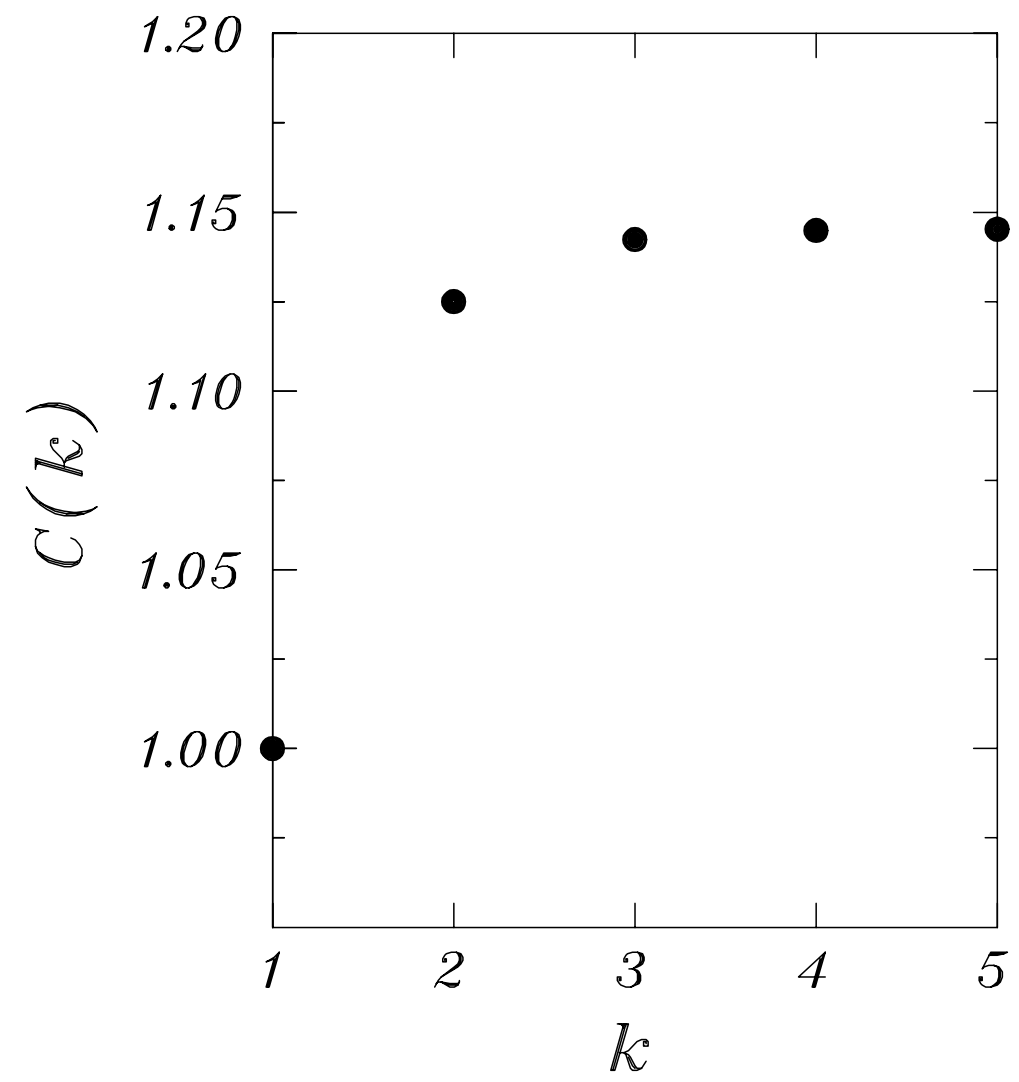

Figure 1. Total cross-sections for the prior and post-prior matrix elements.

From this figure it is evident that the breaking of the post-prior symmetry might have a dramatic effect on the observables. We have explored the consequences of using the interaction also in the $q q$ and $\bar{q} \bar{q}$ channels [34], instead of using it only in the $q \bar{q}$ channel as in Ref. [30]. We obtained the amusing result that the lack of post-prior symmetry is to a large extent compensated by the inclusion of the $q q$ and $\bar{q} \bar{q}$ contributions in our explicitly post-prior symmetrical effective interaction, and the results are consistent with the values obtained in previous phenomenological calculations [30]. 


\section{MAPPING OF BARYONS}

As discussed in the previous sections, there are several appealing features of the Fock-Tani representation that make it particularly interesting for the treatment of composite hadronic interactions in quark models. The extension to the baryon case might be useful for several interesting applications in nuclear physics. The nucleon-nucleon interaction, for example, exhibits a strongly repulsive short-distance core, which is attributed to the exchange of the $\omega$-meson. Since nucleons have a radii of about $0.8 \mathrm{fm}$ and the range of the meson exchange force is $1 / m_{\omega} \approx 0.2 \mathrm{fm}$, it is natural to expect that the nucleon substructure will play a role at such short distances. Other examples include high density and/or temperature nuclear matter where the internal degrees of freedom of hadrons will simultaneously be present with hadronic degrees of freedom.

We start with the definition of the single-composite baryon creation operator, $B_{\alpha}^{\dagger}$, in terms of three quark creation operators

$$
B_{\alpha}^{\dagger}=\frac{1}{\sqrt{3 !}} \Psi_{\alpha}^{\mu_{1} \mu_{2} \mu_{3}} q_{\mu_{1}}^{\dagger} q_{\mu_{2}}^{\dagger} q_{\mu_{3}}^{\dagger} .
$$

$\Psi_{\alpha}^{\mu_{1} \mu_{2} \mu_{3}}$ is the baryon wave function, where the index $\alpha$ identifies the quantum numbers of the baryon, and $\mu$ those of the quarks. The Fock-space amplitude is taken to be orthonormalized,

$$
<\alpha \mid \beta>=\Psi_{\alpha}^{* \mu_{1} \mu_{2} \mu_{3}} \Psi_{\beta}^{\mu_{1} \mu_{2} \mu_{3}}=\delta_{\alpha \beta}
$$

Using the quark anticommutation relations, Eq. (四), and the normalization condition above,

it can easily be shown that the baryon operators satisfy the following noncanonical anticommutation relations

$$
\left\{B_{\alpha}, B_{\beta}^{\dagger}\right\}=\delta_{\alpha \beta}-\Delta_{\alpha \beta}, \quad\left\{B_{\alpha}, B_{\beta}\right\}=0
$$

where

$$
\Delta_{\alpha \beta}=3 \Psi_{\alpha}^{* \mu_{1} \mu_{2} \mu_{3}} \Psi_{\beta}^{\mu_{1} \mu_{2} \nu_{3}} q_{\nu_{3}}^{\dagger} q_{\mu_{3}}-\frac{3}{2} \Psi_{\alpha}^{* \mu_{1} \mu_{2} \mu_{3}} \Psi_{\beta}^{\mu_{1} \nu_{2} \nu_{3}} q_{\nu_{3}}^{\dagger} q_{\nu_{2}}^{\dagger} q_{\mu_{2}} q_{\mu_{3}} .
$$

In addition,

$$
\left\{q_{\mu}, B_{\alpha}^{\dagger}\right\}=\sqrt{\frac{3}{2}} \Psi_{\alpha}^{\mu \mu_{2} \mu_{3}} q_{\mu_{2}}^{\dagger} q_{\mu_{3}}^{\dagger}, \quad\left\{q_{\mu}, B_{\alpha}\right\}=0
$$


As in the case of mesons, the operator $\Delta_{\alpha \beta}$ is due to the composite nature of the baryons.

The unitary transformation is defined such that a single-baryon state $\mid \alpha>$ is transformed into an ("ideal") elementary-baryon state,

$$
\left.\left.\left|\alpha>=B_{\alpha}^{\dagger}\right| 0>\longrightarrow U^{-1}|\alpha>\equiv| \alpha\right)=b_{\alpha}^{\dagger} \mid 0\right)
$$

The ideal baryon operators $b_{\alpha}^{\dagger}$ and $b_{\alpha}$, by definition, satisfy the canonical anticommutation relations

$$
\left\{b_{\alpha}, b_{\beta}^{\dagger}\right\}=\delta_{\alpha \beta}, \quad\left\{b_{\alpha}, b_{\beta}\right\}=0
$$

The state $\mid 0)$ is the vacuum of both $q$ and $b$ degrees of freedom in the new representation. In addition, the quark operators $q^{\dagger}$ and $q$ are kinematically independent of the $b_{\alpha}^{\dagger}$ and $b_{\alpha}$ in the new representation

$$
\left\{q_{\mu}, b_{\alpha}\right\}=\left\{q_{\mu}, b_{\alpha}^{\dagger}\right\}=0
$$

The generator $F$ of the unitary transformation is again an anti-hermitian operator defined by

$$
F=b_{\alpha}^{\dagger} O_{\alpha}-O_{\alpha}^{\dagger} b_{\alpha}
$$

The $O_{\alpha}$ operator is constructed in a iterative manner, order by order, in the baryon wave function by requiring that:

$$
\left\{O_{\alpha}, O_{\beta}^{\dagger}\right\}=\delta_{\alpha \beta}, \quad\left\{O_{\alpha}, O_{\beta}\right\}=\left\{O_{\alpha}^{\dagger}, O_{\beta}^{\dagger}\right\}=0
$$

As in the case for mesons, this iterative procedure ensures that no secular terms appear. The explicit form of the operator $O_{\alpha}$ is obtained in a straightforward calculation, following the same procedure as in the previous section. Up to third order, it is given by

$$
O_{\alpha}=B_{\alpha}+\frac{1}{2} \Delta_{\alpha \beta} B_{\beta}-\frac{1}{2} B_{\beta}^{\dagger}\left[\Delta_{\beta \gamma}, B_{\alpha}\right] B_{\gamma}
$$

It is not difficult to verify that $O_{\alpha}$ has a canonical anticommutation relation up to the third order in the $\Psi$ 's. The next step is to transform the quark operators. 


\section{A. Transformation of the quark operators}

Given the generator $F$ of the baryon transformation to a certain order, one needs to evaluate

$$
\mathcal{O}_{\mathrm{FT}}=U^{-1} \mathcal{O} U
$$

As discussed in the previous section for the mesons, because of Eq. (125), the equations of motion for the operators $b_{\alpha}(t)$ and $O_{\alpha}(t)$ are manifestly symmetric,

$$
\frac{d b_{\alpha}(t)}{d t}=\left[b_{\alpha}(t), F\right]=O_{\alpha}(t), \quad \frac{d O_{\alpha}(t)}{d t}=\left[O_{\alpha}(t), F\right]=-b_{\alpha}(t),
$$

and can be trivially integrated, yielding

$$
b_{\alpha}(t)=O_{\alpha} \sin t+b_{\alpha} \cos t, \quad O_{\alpha}(t)=O_{\alpha} \cos t-b_{\alpha} \sin t .
$$

The transformation of the quark operators follows as in the previous section. However, the transformed operators for the present case contain a few more terms than those for mesons. In order to simplify the presentation the results are quoted for $t=-\pi / 2$.

The zeroth order operator is of course $q_{\mu}^{(0)}=q_{\mu}$. The first order one is

$$
q_{\mu}^{(1)}=\delta_{\mu \mu_{1}} \sqrt{\frac{3}{2}} \Psi_{\beta}^{\mu_{1} \mu_{2} \mu_{3}} q_{\mu_{2}}^{\dagger} q_{\mu_{3}}^{\dagger}\left(b_{\beta}-B_{\beta}\right) .
$$

The solution to the second order equation leads to

$$
\begin{aligned}
q_{\mu}^{(2)} & =\delta_{\mu \nu_{1}} \frac{3}{2} \Psi_{\alpha}^{* \mu_{1} \mu_{2} \mu_{3}}\left[\Psi_{\beta}^{\nu_{1} \mu_{2} \mu_{3}}\left(b_{\alpha}^{\dagger} q_{\mu_{1}} b_{\beta}+B_{\alpha}^{\dagger} q_{\mu_{1}} B_{\beta}-2 B_{\alpha}^{\dagger} q_{\mu_{1}} b_{\beta}\right)\right. \\
& \left.-\Psi_{\beta}^{\nu_{1} \nu_{2} \mu_{3}}\left(b_{\alpha}^{\dagger} q_{\nu_{2}}^{\dagger} q_{\mu_{2}} q_{\mu_{3}} b_{\beta}+B_{\alpha}^{\dagger} q_{\nu_{2}}^{\dagger} q_{\mu_{2}} q_{\mu_{3}} B_{\beta}-2 B_{\alpha}^{\dagger} q_{\nu_{2}}^{\dagger} q_{\mu_{2}} q_{\mu_{3}} b_{\beta}\right)\right] .
\end{aligned}
$$

And finally, the solution of the third order equation is

$$
\begin{aligned}
& q_{\mu}^{(3)}=\delta_{\mu \sigma_{1}} \sqrt{\frac{3}{2}}\left\{\frac{1}{2} \Psi_{\alpha}^{\sigma_{1} \mu_{2} \mu_{3}} q_{\mu_{2}}^{\dagger} q_{\mu_{3}}^{\dagger} \Delta_{\alpha \gamma}\left(b_{\gamma}-2 B_{\gamma}\right)\right. \\
& +\frac{3}{2} \Psi_{\alpha}^{* \mu_{1} \mu_{2} \mu_{3}}\left[\Psi_{\beta}^{* \nu_{1} \nu_{2} \nu_{3}} \Psi_{\gamma}^{\sigma_{1} \mu_{2} \nu_{3}}\left(B_{\alpha}^{\dagger}-b_{\alpha}^{\dagger}\right) B_{\beta}^{\dagger} q_{\mu_{1}} q_{\mu_{3}} q_{\nu_{2}} q_{\nu_{1}} b_{\gamma}\right. \\
& +\left(2 \Psi_{\beta}^{\mu_{1} \mu_{2} \nu_{3}} \Psi_{\gamma}^{\sigma_{1} \nu_{2} \mu_{3}}-\Psi_{\beta}^{\mu_{1} \nu_{2} \nu_{3}} \Psi_{\gamma}^{\sigma_{1} \mu_{2} \mu_{3}}\right) q_{\nu_{2}}^{\dagger} q_{\nu_{3}}^{\dagger}\left(-b_{\alpha}^{\dagger} b_{\beta} b_{\gamma}+B_{\alpha}^{\dagger} B_{\beta} B_{\gamma}-B_{\alpha}^{\dagger} B_{\beta} b_{\gamma}+b_{\alpha}^{\dagger} B_{\beta} b_{\gamma}\right) \\
& \left.\left.+2 \Psi_{\beta}^{\nu_{1} \mu_{2} \nu_{3}} \Psi_{\gamma}^{\sigma_{1} \nu_{2} \mu_{3}} q_{\nu_{1}}^{\dagger} q_{\nu_{2}}^{\dagger} q_{\nu_{3}}^{\dagger}\left(-b_{\alpha}^{\dagger} b_{\beta} b_{\gamma}+B_{\alpha}^{\dagger} B_{\beta} B_{\gamma}-B_{\alpha}^{\dagger} B_{\beta} b_{\gamma}+b_{\alpha}^{\dagger} B_{\beta} b_{\gamma}\right) q_{\mu_{1}}\right]\right\} .
\end{aligned}
$$

This result concludes the evaluation of the transformed operators which will be necessary to construct the Hamiltonian in the new representation. This is done in the next section. 


\section{B. Effective baryon Hamiltonian}

The Fock-Tani Hamiltonian $H_{\mathrm{FT}}$ is obtained from the quark-quark part of Hamiltonian in Eq. (55). The free-space eigenvalue equation for the single-baryon state is

$$
H(\mu \nu ; \sigma \rho) \Psi_{\alpha}^{\sigma \rho \lambda}=\mathcal{E}_{[\alpha]} \Psi_{[\alpha]}^{\mu \nu \lambda}
$$

where the Hamiltonian matrix $H(\mu \nu ; \sigma \rho)$ is given by

$$
H(\mu \nu, \sigma \rho)=3\left[\delta_{[\mu] \sigma} \delta_{\nu \rho} T([\mu])+V_{q q}(\mu \nu ; \sigma \rho)\right]
$$

$\mathcal{E}_{\alpha}$ is the total energy of the baryon. Here the notation is once again used so that there is no summation over repeated indices in square brackets.

The Fock-Tani Hamiltonian for baryons has a general structure similar to the $H_{\mathrm{FT}}$ of mesons, namely,

$$
H_{\mathrm{FT}}=H_{q}+H_{b}+H_{b q}
$$

The term $H_{q}$ involves only quark operators, $H_{b}$ only ideal baryon operators, and $H_{b q}$ involves both quark and ideal baryon operators.

In order to obtain the quark-quark interaction in the new representation, only the appropriate terms need be collected from the lowest order ones $q_{\mu}^{\dagger(0)} q_{\mu}^{(1)}$ and $q_{\mu}^{\dagger(1)} q_{\mu}^{(1)}$, from the transformation of kinetic energy, and $q_{\mu}^{\dagger(1)} q_{\nu}^{\dagger(0)} q_{\rho}^{(0)} q_{\sigma}^{(0)}$ and $q_{\mu}^{\dagger(1)} q_{\nu}^{\dagger(0)} q_{\rho}^{(0)} q_{\sigma}^{(1)}$, from the transformation of the potential. This leads for the new quark-quark interaction the following expression

$$
\begin{aligned}
V_{q q}= & \frac{1}{2} V_{q q}(\mu \nu ; \sigma \rho) q_{\mu}^{\dagger} q_{\nu}^{\dagger} q_{\rho} q_{\sigma} \\
& +\frac{1}{6}\left[\Delta\left(\nu_{1} \nu_{2} \nu_{3} ; \mu_{1} \mu \nu\right) H\left(\mu \nu ; \mu_{2} \mu_{3}\right)+H\left(\nu_{1} \nu_{2} ; \sigma \rho\right) \Delta\left(\sigma \rho \nu_{3} ; \mu_{1} \mu_{2} \mu_{3}\right)\right. \\
& \left.-\Delta\left(\nu_{1} \nu_{2} \nu_{3} ; \tau \mu \nu\right) H(\mu \nu ; \sigma \rho) \Delta\left(\sigma \rho \tau ; \mu_{1} \mu_{2} \mu_{3}\right)\right] q_{\nu_{1}}^{\dagger} q_{\nu_{2}}^{\dagger} q_{\nu_{3}}^{\dagger} q_{\mu_{1}} q_{\mu_{2}} q_{\mu_{3}},
\end{aligned}
$$

where $\Delta\left(\mu \nu \tau ; \mu^{\prime} \nu^{\prime} \tau^{\prime}\right)$ is the bound state kernel for baryons,

$$
\Delta\left(\mu \nu \tau ; \mu^{\prime} \nu^{\prime} \tau^{\prime}\right)=\sum_{\alpha} \Psi_{\alpha}^{\mu \nu \tau} \Psi_{\alpha}^{* \mu^{\prime} \nu^{\prime} \tau^{\prime}}
$$


In the case that the $\Psi$ 's are chosen to be the eigenstates of the microscopic quark Hamiltonian, Eq. (136) can be written in the following suggestive way

$$
V_{q q}=\frac{1}{2} V_{q q}(\mu \nu ; \sigma \rho) q_{\mu}^{\dagger} q_{\nu}^{\dagger} q_{\rho} q_{\sigma}-\sum_{\alpha} \mathcal{E}_{\alpha} B_{\alpha}^{\dagger} B_{\alpha} .
$$

As in the case of mesons, it is not difficult to show that the spectrum of the modified quark Hamiltonian $H_{q}$ in Eq. (135) is positive semidefinite.

Note that because $V_{q q}(\mu \nu ; \sigma \rho)$ is a two-body interaction, the creation and annihilation operators with sub-index 3 can always be contracted with each other in Eq. (136), such that the new quark-quark interaction, $V_{q q}$, remains in a two-body form .

Among the various baryon-quark Hamiltonians $H_{b q}$ in Eq. (135), the two interesting ones are the single-baryon break-up and the binary collision break-up. By collecting the appropriate lowest order terms of the transformation of the kinetic and potential terms, the single-baryon break-up interaction (single-break) is obtained as

$$
\begin{aligned}
H_{\text {single-break }} & =\frac{1}{\sqrt{6}}\left[H\left(\mu_{1} \mu_{2} ; \sigma \rho\right) \Psi_{\beta}^{\sigma \rho \mu_{3}}\right. \\
& \left.-H(\mu \nu ; \sigma \rho) \Psi_{\beta}^{\sigma \rho \tau_{3}} \Delta\left(\mu_{1} \mu_{2} \mu_{3} ; \mu \nu \tau_{3}\right)\right] q_{\mu_{1}}^{\dagger} q_{\mu_{2}}^{\dagger} q_{\mu_{3}}^{\dagger} b_{\beta} .
\end{aligned}
$$

The hermitian conjugate of $H_{\text {single-break }}$ represents a baryon recombination. When $\Psi$ is chosen to be a stationary state of the microscopic quark Hamiltonian, Eq. (139) becomes

$$
H_{\text {single-break }}=0 .
$$

This result reflects the stability of the baryon state to spontaneous decay in the absence of external perturbations. For a baryon in the environment produced in a heavy-ion collision, for example, one would be interested in the modifications on the free-space wave function properties of the nucleons, and obviously the effective Hamiltonian of Eq. (139) would be relevant in such a situation.

In the same way, a binary baryon collision break-up (binary-break) term can be found,

$$
\begin{aligned}
V_{\text {binary-break }} & =\frac{1}{2} V_{\mathrm{qq}}(\mu \nu ; \sigma \rho) q_{\mu}^{\dagger(0)} q_{\nu}^{\dagger(0)} q_{\rho}^{(1)} q_{\sigma}^{(1)} \\
& =\frac{3}{4} \Psi_{\alpha}^{\rho \nu_{2} \mu_{3}} \Psi_{\beta}^{\sigma \nu_{2} \nu_{3}} V_{q q}(\mu \nu ; \sigma \rho) q_{\mu}^{\dagger} q_{\nu}^{\dagger} q_{\mu_{2}}^{\dagger} q_{\mu_{3}}^{\dagger} q_{\nu_{2}}^{\dagger} q_{\nu_{3}}^{\dagger} b_{\alpha} b_{\beta} .
\end{aligned}
$$

In a hot and/or dense medium, where constituents and composites can be simultaneously present in the system break-up terms may play an important role. 
The piece of the effective Hamiltonian that contains only ideal baryon operators, represented by the second term of Eq. (135), can be written as:

$$
H_{b}=H_{b}^{(0)}+V_{b b}
$$

$H_{b b}^{(0)}$ is the "non-interacting" term, obtained by substituting the transformed operators into the expression

$$
T(\mu) q_{\mu}^{\dagger(1)}(t) q_{\mu}^{(1)}(t)+\frac{1}{2} V_{q q}(\mu \nu ; \sigma \rho) q_{\mu}^{\dagger(1)}(t) q_{\nu}^{\dagger(0)}(t) q_{\rho}^{(0)}(t) q_{\sigma}^{(1)}(t)
$$

After putting the expression in normal order, a term is obtained that contains only ideal baryon operators,

$$
H_{b}^{(0)}=H_{\mathrm{FT}}^{(0)}(\alpha ; \beta) b_{\alpha}^{\dagger} b_{\beta}
$$

where

$$
H_{\mathrm{FT}}^{(0)}(\alpha ; \beta)=\Psi_{\alpha}^{* \mu \nu \tau} H(\mu \nu ; \sigma \rho) \Psi_{\beta}^{\sigma \rho \tau}
$$

The baryon-baryon potential is obtained from the expression

$$
\begin{aligned}
& T(\mu)\left[q_{\mu}^{\dagger(3)}(t) q_{\mu}^{(1)}(t)+q_{\mu}^{\dagger(1)}(t) q_{\mu}^{(3)}(t)\right]+\frac{1}{2} V_{q q}(\mu \nu ; \sigma \rho)\left[q_{\mu}^{\dagger(1)}(t) q_{\nu}^{\dagger(1)}(t) q_{\rho}^{(1)}(t) q_{\sigma}^{(1)}(t)\right. \\
& +q_{\mu}^{\dagger(1)}(t) q_{\nu}^{\dagger(0)}(t) q_{\rho}^{(2)}(t) q_{\sigma}^{(1)}(t)+q_{\mu}^{\dagger(1)}(t) q_{\nu}^{\dagger(2)}(t) q_{\rho}^{(0)}(t) q_{\sigma}^{(1)}(t) \\
& \left.+q_{\mu}^{\dagger(3)}(t) q_{\nu}^{\dagger(0)}(t) q_{\rho}^{(0)}(t) q_{\sigma}^{(1)}(t)+q_{\mu}^{\dagger(1)}(t) q_{\nu}^{\dagger(0)}(t) q_{\rho}^{(0)}(t) q_{\sigma}^{(3)}(t)\right] .
\end{aligned}
$$

Substituting the transformed quark operators, and putting the resulting expression in normal order, terms are obtained that again involve only ideal baryon operators. The total baryon Hamiltonian may be written in the form

$$
H_{b}=\Psi_{\alpha}^{* \mu \nu \lambda} H(\mu \nu ; \sigma \rho) \Psi_{\beta}^{\sigma \rho \lambda} b_{\alpha}^{\dagger} b_{\beta}+\frac{1}{2} V_{b b}(\alpha \beta ; \delta \gamma) b_{\alpha}^{\dagger} b_{\beta}^{\dagger} b_{\gamma} b_{\delta}
$$

where $V_{b b} \equiv V_{b b}^{d i r}+V_{b b}^{e x c}+V_{b b}^{i n t}$ is the effective baryon-baryon potential, which is divided we divide again into direct, exchange, and intra-exchange parts, where 


$$
\begin{aligned}
V_{b b}^{d i r}(\alpha \beta ; \delta \gamma)= & 9 V_{q q}(\mu \nu ; \sigma \rho) \Psi_{\alpha}^{* \mu \mu_{2} \mu_{3}} \Psi_{\beta}^{* \nu \nu_{2} \nu_{3}} \Psi_{\gamma}^{\rho \nu_{2} \nu_{3}} \Psi_{\delta}^{\sigma \mu_{2} \mu_{3}} \\
V_{b b}^{e x c}(\alpha \beta ; \delta \gamma)= & 9 V_{q q}(\mu \nu ; \sigma \rho)\left[2 \Psi_{\alpha}^{* \mu \nu \mu_{3}} \Psi_{\beta}^{* \nu_{1} \nu_{2} \nu_{3}} \Psi_{\gamma}^{\rho \nu_{2} \nu_{3}} \Psi_{\delta}^{\nu_{1} \sigma \mu_{3}}\right. \\
& -\Psi_{\alpha}^{* \mu \mu_{2} \mu_{3}} \Psi_{\beta}^{* \nu \nu_{2} \nu_{3}}\left(\Psi_{\gamma}^{\sigma \nu_{2} \nu_{3}} \Psi_{\delta}^{\rho \mu_{2} \mu_{3}}+4 \Psi_{\gamma}^{\rho \nu_{2} \mu_{3}} \Psi_{\delta}^{\sigma \mu_{2} \nu_{3}}\right) \\
& \left.-2 \Psi_{\alpha}^{* \mu \mu_{2} \mu_{3}} \Psi_{\beta}^{* \nu_{1} \nu_{2} \nu} \Psi_{\gamma}^{\nu_{1} \nu_{2} \mu_{3}} \Psi_{\delta}^{\sigma \mu_{2} \rho}\right] \\
V_{b b}^{i n t}(\alpha \beta ; \delta \gamma)= & -6 H(\mu \nu ; \sigma \rho)\left(\Psi_{\alpha}^{* \mu \nu \mu_{3}} \Psi_{\beta}^{* \nu_{1} \nu_{2} \nu_{3}} \Psi_{\gamma}^{\nu_{1} \nu_{2} \mu_{3}} \Psi_{\delta}^{\sigma \rho \nu_{3}}\right. \\
& \left.+\Psi_{\alpha}^{* \mu \mu_{2} \nu} \Psi_{\beta}^{* \nu_{1} \nu_{2} \nu_{3}} \Psi_{\gamma}^{\nu_{1} \nu_{2} \rho} \Psi_{\delta}^{\sigma \mu_{2} \nu_{3}}+\Psi_{\alpha}^{* \mu \mu_{2} \mu_{3}} \Psi_{\beta}^{* \nu_{1} \nu_{2} \nu^{\nu}} \Psi_{\gamma}^{\nu_{1} \nu_{2} \mu_{3}} \Psi_{\delta}^{\sigma \mu_{2} \rho}\right) .
\end{aligned}
$$

Similar to the meson case, it can be shown that if the $\Psi$ 's are chosen to be the eigenstates of the microscopic quark Hamiltonian, the intra-exchange term $V_{b b}^{i n t}$ is precisely canceled by orthogonality corrections at lowest order.

In the next subsection, our nucleon-nucleon result is compared with the one obtained in Ref. [32. In the quark model used in Ref. [32], the $\Psi$ 's are taken to be nonrelativistic s-wave gaussians, and the microscopic quark-quark interaction is the spin-spin part of the nonrelativistic reduction of the one-gluon exchange.

\section{An effective nucleon-nucleon potential}

According to our notation, the nucleon wave function used in Ref. [32] is given by

$$
\Psi_{\alpha}^{\mu_{1} \mu_{2} \mu_{3}} \equiv \delta\left(\boldsymbol{p}_{\alpha}-\boldsymbol{p}_{1}-\boldsymbol{p}_{2}-\boldsymbol{p}_{3}\right) \mathcal{N}\left(\boldsymbol{p}_{\alpha}\right) \frac{\epsilon^{c_{\mu_{1}} c_{\mu_{2}} c_{\mu_{3}}}}{\sqrt{3 !}} \epsilon^{c_{\mu_{1}} c_{\mu_{2}} c_{\mu_{3}}} \chi_{\alpha}^{I_{\mu_{1}} I_{\mu_{2}} I_{\mu_{3}}} \phi\left(\boldsymbol{p}_{1}\right) \phi\left(\boldsymbol{p}_{2}\right) \phi\left(\boldsymbol{p}_{3}\right),
$$

where $\epsilon^{c_{\mu_{1}} c_{\mu_{2}} c_{\mu_{3}}}$ is the color antisymmetric tensor; $\chi_{\alpha}^{I_{\mu_{1}} I_{\mu_{2}} I_{\mu_{3}}}$ is the Clebsch-Gordan coefficient of spin-isospin, the $\phi$ 's are the single-quark wave functions, and $\mathcal{N}\left(\boldsymbol{p}_{\alpha}\right)$ is a normalization function. The explicit form of the momentum-space single-quark functions is

$$
\phi(\boldsymbol{p})=\left(\frac{b^{2}}{\pi}\right)^{3 / 4} \exp \left(-\frac{b^{2} \boldsymbol{p}^{2}}{2}\right)
$$

With such a form, $\mathcal{N}(\boldsymbol{p})$ is obtained to be

$$
\mathcal{N}(\boldsymbol{p})=\left(\frac{3 \pi}{b^{2}}\right)^{3 / 4} \exp \left(\frac{b^{2} \boldsymbol{p}^{2}}{6}\right) .
$$

The constant $b$ is related to the mean-square radius of the baryon by $b^{2}=<r^{2}>$.

Using a local quark-quark interaction $V_{q q}$ as in Ref. [32, i.e. an interaction that depends only on the momentum transfer, the 12-dimensional integral over the quark coordinates in 
the expression of the effective potential can be evaluated analytically, because the singlequark wave functions are gaussians. Then the NN potential can be written in the form

$$
\begin{aligned}
V_{N N} & =\frac{1}{2} \int d \boldsymbol{p}_{1} \cdots d \boldsymbol{p}_{4} \delta\left(\boldsymbol{p}_{1}+\boldsymbol{p}_{2}-\boldsymbol{p}_{3}-\boldsymbol{p}_{4}\right)<\lambda_{3} \lambda_{4}\left|V_{N N}\left(\boldsymbol{\sigma}, \boldsymbol{\tau}, \boldsymbol{p}_{1} \cdots \boldsymbol{p}_{4}\right)\right| \lambda_{1} \lambda_{2}> \\
& \times b_{\lambda_{4}}^{\dagger}\left(\boldsymbol{p}_{4}\right) b_{\lambda_{3}}^{\dagger}\left(\boldsymbol{p}_{3}\right) b_{\lambda_{2}}\left(\boldsymbol{p}_{2}\right) b_{\lambda_{1}}\left(\boldsymbol{p}_{1}\right),
\end{aligned}
$$

where $\lambda=\left(M_{S}, M_{T}\right)$ and

$$
V_{N N}\left(\boldsymbol{\sigma}, \boldsymbol{\tau}, \boldsymbol{p}_{1} \cdots \boldsymbol{p}_{4}\right)=\sum_{i=1}^{5} \omega_{i}(\boldsymbol{\sigma}, \boldsymbol{\tau}) v_{i}\left(\boldsymbol{p}_{1} \cdots \boldsymbol{p}_{4}\right) .
$$

The operators $\omega_{i}(\boldsymbol{\sigma}, \boldsymbol{\tau})$ are obtained from the sum over the quark color-spin-flavor indices; $\boldsymbol{\sigma}_{N}$ and $\boldsymbol{\tau}_{N}$ are nucleon spin and isospin operators. The sum over the quark spin-isospin quantum numbers can be evaluated in closed form by making use of the elegant technique of Ref. 35. The spatial functions can be written as

$$
\begin{aligned}
& v_{1}\left(\boldsymbol{p}_{1} \cdots \boldsymbol{p}_{4}\right)=F\left(\boldsymbol{q}^{2}\right) V_{\mathrm{qq}}(\boldsymbol{q}) F\left(\boldsymbol{q}^{2}\right), \\
& v_{i}\left(\boldsymbol{p}_{1} \cdots \boldsymbol{p}_{4}\right)=\left(\frac{3 b^{2}}{4 \pi}\right)^{3 / 2} N\left(\boldsymbol{p}_{1} \cdots \boldsymbol{p}_{4}\right) I_{i}\left(\boldsymbol{p}_{1} \cdots \boldsymbol{p}_{4}\right),
\end{aligned}
$$

where $\boldsymbol{q}=\boldsymbol{p}_{3}-\boldsymbol{p}_{1}=\boldsymbol{p}_{2}-\boldsymbol{p}_{4}$ is the momentum transfer, $F\left(\boldsymbol{q}^{2}\right)$ is the nucleon form factor,

$$
F\left(\boldsymbol{q}^{2}\right)=\exp \left(-\frac{b^{2}}{6} \boldsymbol{q}^{2}\right)
$$

and $N\left(\boldsymbol{p}_{1} \cdots \boldsymbol{p}_{4}\right)$ is given by

$$
N\left(\boldsymbol{p}_{1} \cdots \boldsymbol{p}_{4}\right)=\exp \left[-b^{2}\left(\frac{1}{2} \boldsymbol{p}_{4}^{2}+\frac{1}{4} \boldsymbol{p}_{3}^{2}+\frac{7}{12} \boldsymbol{p}_{2}^{2}-\frac{1}{6} \boldsymbol{p}_{1}^{2}-\boldsymbol{p}_{4} \cdot \boldsymbol{p}_{2}-\frac{1}{3} \boldsymbol{p}_{3} \cdot \boldsymbol{p}_{2}+\frac{1}{3} \boldsymbol{p}_{4} \cdot \boldsymbol{p}_{3}\right)\right],
$$

and $I_{i}\left(\boldsymbol{p}_{1} \cdots \boldsymbol{p}_{4}\right), i=2, \cdots, 5$ are the integrals

$$
\begin{aligned}
& I_{2}\left(\boldsymbol{p}_{1} \cdots \boldsymbol{p}_{4}\right)=\int d \boldsymbol{q} V_{\mathrm{qq}}(\boldsymbol{q}) \exp \left[-b^{2}\left(q^{2}-\boldsymbol{q} \cdot\left(\boldsymbol{p}_{1}-\boldsymbol{p}_{2}\right)\right)\right], \\
& I_{3}\left(\boldsymbol{p}_{1} \cdots \boldsymbol{p}_{4}\right)=\int d \boldsymbol{q} V_{\mathrm{qq}}(\boldsymbol{q}) \exp \left[-b^{2}\left(\frac{3}{4} \boldsymbol{q}^{2}+\frac{1}{2} \boldsymbol{q} \cdot\left(\boldsymbol{p}_{3}-\boldsymbol{p}_{2}\right)\right)\right], \\
& I_{4}\left(\boldsymbol{p}_{4} \cdots \boldsymbol{p}_{4}\right)=\int d \boldsymbol{q} V_{\mathrm{qq}}(\boldsymbol{q}) \exp \left[-b^{2}\left(\frac{11}{16} \boldsymbol{q}^{2}+\boldsymbol{q} \cdot\left(\frac{1}{2} \boldsymbol{p}_{4}+\frac{1}{4} \boldsymbol{p}_{3}-\frac{3}{4} \boldsymbol{p}_{2}\right)\right)\right], \\
& I_{5}\left(\boldsymbol{p}_{1} \cdots \boldsymbol{p}_{4}\right)=\int d \boldsymbol{q} V_{\mathrm{qq}}(\boldsymbol{q}) \exp \left[-b^{2}\left(\frac{11}{16} \boldsymbol{q}^{2}-\boldsymbol{q} \cdot\left(\frac{1}{2} \boldsymbol{p}_{4}-\frac{1}{4} \boldsymbol{p}_{3}-\frac{1}{4} \boldsymbol{p}_{2}\right)\right)\right] .
\end{aligned}
$$

When using $V_{q q}(\boldsymbol{q})=V_{0}=$ constant, which represents a contact interaction, such as the spin-spin term from the one-gluon exchange used in Ref. [32], the following expressions for the $v_{i}$ 's are obtained 


$$
\begin{aligned}
& v_{1}\left(\boldsymbol{p}, \boldsymbol{p}^{\prime}\right)=v_{3}\left(\boldsymbol{p}, \boldsymbol{p}^{\prime}\right)=V_{0} \exp \left[-\frac{b^{2}}{3}\left(\boldsymbol{p}-\boldsymbol{p}^{\prime}\right)^{2}\right] \\
& v_{2}\left(\boldsymbol{p}, \boldsymbol{p}^{\prime}\right)=V_{0}\left(\frac{3}{4}\right)^{3 / 2} \exp \left[-\frac{b^{2}}{6}\left(\boldsymbol{p}^{2}+\boldsymbol{p}^{\prime 2}\right)\right] \\
& v_{4}\left(\boldsymbol{p}, \boldsymbol{p}^{\prime}\right)=v_{5}\left(\boldsymbol{p}^{\prime}, \boldsymbol{p}\right)=V_{0}\left(\frac{12}{11}\right)^{3 / 2} \exp \left[-\frac{2 b^{2}}{11}\left(\boldsymbol{p}-\boldsymbol{p}^{\prime}\right)^{2}-\frac{b^{2}}{33}\left(\boldsymbol{p}^{2}+7 \boldsymbol{p}^{\prime 2}\right)\right] .
\end{aligned}
$$

Now, when the color-spin-flavor dependence of the interaction is equal to the spin-spin term of the one-gluon exchange, Eq. (95), the spin-isospin functions $\omega_{i}(\boldsymbol{\sigma}, \boldsymbol{\tau})$ are given by

$$
\begin{aligned}
& \omega_{1}=0 \\
& \omega_{2}=\frac{1}{12}\left[\left(1+\frac{1}{9} \boldsymbol{\tau}_{N}^{1} \cdot \boldsymbol{\tau}_{N}^{2}\right)+\frac{1}{3}\left(1+\frac{1}{9} \boldsymbol{\tau}_{N}^{1} \cdot \boldsymbol{\tau}_{N}^{2}\right) \boldsymbol{\sigma}_{N}^{1} \cdot \boldsymbol{\sigma}_{N}^{2}\right], \\
& \omega_{3}=\frac{3}{4}\left[\left(1+\frac{1}{9} \boldsymbol{\tau}_{N}^{1} \cdot \boldsymbol{\tau}_{N}^{2}\right)-\frac{1}{27}\left(1+\frac{25}{9} \boldsymbol{\tau}_{N}^{1} \cdot \boldsymbol{\tau}_{N}^{2}\right) \boldsymbol{\sigma}_{N}^{1} \cdot \boldsymbol{\sigma}_{N}^{2}\right], \\
& \omega_{4}=\omega_{5}=\frac{1}{4}\left[\left(1-\frac{1}{9} \boldsymbol{\tau}_{N}^{1} \cdot \boldsymbol{\tau}_{N}^{2}\right)-\frac{1}{9}\left(1-\frac{5}{9} \boldsymbol{\tau}_{N}^{1} \cdot \boldsymbol{\tau}_{N}^{2}\right) \boldsymbol{\sigma}_{N}^{1} \cdot \boldsymbol{\sigma}_{N}^{2}\right],
\end{aligned}
$$

Note that $\omega_{1}=0$ because there is no one-gluon exchange between colorless baryons.

On-shell, i.e., when $\boldsymbol{p}^{2}=\boldsymbol{p}^{\prime 2}$, this result is precisely the same as obtained within the quark Born diagram method for the T-matrix [32]. For the off-shell case, it is evident that our interaction is post-prior symmetric. The symmetry is of importance for calculations beyond the Born approximation, where the potential is iterated in a Lippmann-Schwinger equation.

In the next section orthogonality corrections to the lowest order hadron-hadron Hamiltonians will be derived. The study of the hadron-hadron interaction using the constituent quark model has been traditionally been done with the resonating group method (RGM). There is an extensive literature on the subject; two good review articles are given in Ref. [36]. The RGM will be used in the next section to make contact with the Fock-Tani representation, and to illustrate in a transparent way the physical meaning of the orthogonality corrections for the effective meson-meson interaction. 


\section{ORTHOGONALITY CORRECTIONS}

Orthogonality corrections appear in the form of terms proportional to the bound state kernels $\Delta(\mu \nu ; \sigma \rho)$ and $\Delta(\mu \nu \lambda ; \sigma \rho \tau)$, and have the effect, among others, of weakening the "intra-exchange" interactions. An example of this effect is the renormalization of the microscopic interaction, shown in Eqs. (59) and (136). In the hadron-hadron interaction, they reflect the Pauli principle among the constituents in the different clusters. In this section, the lowest-order corrections (in an expansion in powers of the bound state kernels $\Delta$ ) for the effective meson and baryon Hamiltonians are obtained, and the RGM is used to evaluate the magnitude of higher oder terms.

Before entering into the derivation of the orthogonality corrections within the FockTani representation, use is made of the RGM for the meson-meson scattering. The RGM is extensively used in the context of hadron-hadron scattering [36], and the meaning and origin of these corrections is particularly transparent within this method. Of course, as will become clear at the end of the discussion, orthogonality corrections apply to all pieces of the effective Hamiltonian, not only to the effective hadron-hadron interaction, and can be systematically derived from within the Fock-Tani representation.

In a RGM calculation the two-cluster state is introduced by writing

$$
\left|\Lambda>=\frac{1}{\sqrt{2}} \psi_{\Lambda}^{\alpha \beta} M_{\alpha}^{\dagger} M_{\beta}^{\dagger}\right| 0>,
$$

where $\psi_{\Lambda}^{\alpha \beta}$ is the ansatz wave function for the meson pair; it describes the c.m. and relative motions of the two meson clusters. The $M^{\dagger}$ 's are the meson creation operators as defined in Eq. (21). $\Lambda$ identifies the set of quantum numbers of the two-cluster state. Using the commutation relation of the meson operators, Eq. (5), the normalization condition for the $\psi_{\Lambda}^{\alpha \beta}$ is obtained to be

$$
<\Lambda \mid \Lambda^{\prime}>=\psi_{\Lambda}^{* \alpha \beta} N\left(\alpha \beta ; \alpha^{\prime} \beta^{\prime}\right) \psi_{\Lambda^{\prime}}^{\alpha^{\prime} \beta^{\prime}}=\delta_{\Lambda^{\prime} \Lambda}
$$

where $N\left(\alpha \beta ; \alpha^{\prime} \beta^{\prime}\right)$ is the "normalization kernel", given by

$$
N\left(\alpha \beta ; \alpha^{\prime} \beta^{\prime}\right)=\delta_{\alpha \alpha^{\prime}} \delta_{\beta \beta^{\prime}}-N_{E}\left(\alpha \beta ; \alpha^{\prime} \beta^{\prime}\right)=\delta_{\alpha \alpha^{\prime}} \delta_{\beta \beta^{\prime}}-\Phi_{\alpha}^{* \mu \nu} \Phi_{\beta}^{* \rho \sigma} \Phi_{\beta^{\prime}}^{\mu \sigma} \Phi_{\alpha^{\prime}}^{\rho \nu}
$$

The exchange kernel $N_{E}\left(\alpha \beta ; \alpha^{\prime} \beta^{\prime}\right)$ comes from the noncanonical part of the meson commutation relation of Eq. (5), and it reflects the Pauli principle among the quarks and antiquarks 
in the clusters $\alpha$ and $\beta$. The equation of motion for $\psi_{\Lambda}^{\alpha \beta}$ is determined by means of the variational principle

$$
\delta<\Lambda\left|\left(H-E_{\Lambda}\right)\right| \Lambda>=0
$$

which leads to the RGM equation,

$$
\left[H_{R G M}(\alpha \beta ; \gamma \delta)-E_{\Lambda} N(\alpha \beta ; \gamma \delta)\right] \psi_{\Lambda}^{\gamma \delta}=0
$$

with

$$
H_{R G M}(\alpha \beta ; \gamma \delta)=T_{R G M}(\alpha \beta ; \gamma \delta)+V_{m m}(\alpha \beta ; \gamma \delta)
$$

where the kinetic term $T_{R G M}(\alpha \beta ; \gamma \delta)$ is given by

$$
T_{R G M}(\alpha \beta ; \gamma \delta)=\delta_{\beta \delta} \Phi_{\alpha}^{* \mu \nu} H\left(\mu \nu ; \mu^{\prime} \nu^{\prime}\right) \Phi_{\gamma}^{\mu^{\prime} \nu^{\prime}}+\delta_{\alpha \gamma} \Phi_{\beta}^{* \mu \nu} H\left(\mu \nu ; \mu^{\prime} \nu^{\prime}\right) \Phi_{\delta}^{\mu^{\prime} \nu^{\prime}}
$$

and the potential terms $V_{m m}(\alpha \beta ; \gamma \delta)$ are precisely equal to the Fock-Tani potentials given in Eqs. (66)-(69).

The two-meson wave function is not normalized in the usual quantum mechanical way, because of the presence of normalization kernel in Eq. (167). It is common practice [36] to introduce a "renormalized" wave function defined as

$$
\bar{\psi}_{\Lambda}^{\alpha \beta} \equiv N^{\frac{1}{2}}\left(\alpha \beta ; \alpha^{\prime} \beta^{\prime}\right) \psi_{\Lambda}^{\alpha^{\prime} \beta^{\prime}}
$$

where $N^{1 / 2}$ is the square root of the RGM normalization kernel. This clearly leads to

$$
\bar{\psi}_{\Lambda^{\prime}}^{* \alpha \beta} \bar{\psi}_{\Lambda}^{\alpha \beta}=\delta_{\Lambda^{\prime} \Lambda}
$$

In terms of the renormalized wave function, the RGM equation can be rewritten as

$$
\left[\bar{H}_{R G M}(\alpha \beta ; \gamma \delta)-E_{\Lambda} \delta_{\alpha \gamma} \delta_{\beta \delta}\right] \bar{\psi}_{\Lambda}^{\gamma \delta}=0
$$

where the "renormalized" RGM Hamiltonian is defined as

$$
\bar{H}_{R G M}(\alpha \beta ; \gamma \delta) \equiv N^{-\frac{1}{2}}\left(\alpha \beta ; \alpha^{\prime} \beta^{\prime}\right) H_{R G M}\left(\alpha^{\prime} \beta^{\prime} ; \gamma^{\prime} \delta^{\prime}\right) N^{-\frac{1}{2}}\left(\gamma^{\prime} \delta^{\prime} ; \gamma \delta\right)
$$

Now, if $N^{-\frac{1}{2}}$ is expanded in Eq. (176) according to 


$$
N^{-\frac{1}{2}}=\left(1-N_{E}\right)^{-\frac{1}{2}}=1+\frac{1}{2} N_{E}+\frac{3}{8} N_{E}^{2}+\cdots,
$$

where $N_{E}$ is the exchange kernel defined in Eq. (168), and only the first order term is retained, the lowest order correction to the RGM Hamiltonian is given by

$$
\begin{aligned}
& \bar{H}_{R G M}(\alpha \beta ; \gamma \delta)=T_{R G M}(\alpha \beta ; \gamma \delta)+V_{m m}^{d i r}(\alpha \beta ; \gamma \delta)+V_{m m}^{e x c}(\alpha \beta ; \gamma \delta) \\
& \quad-\frac{1}{2}\left\{\Phi_{\alpha}^{* \mu \nu} \Phi_{\beta}^{* \rho \sigma}\left[H\left(\mu \nu ; \mu^{\prime} \nu^{\prime}\right)-H(\mu \nu ; \lambda \tau) \Delta\left(\lambda \tau ; \mu^{\prime} \nu^{\prime}\right)\right] \Phi_{\delta}^{\mu^{\prime} \sigma} \Phi_{\gamma}^{\rho \nu^{\prime}}+(\alpha \leftrightarrow \beta ; \gamma \leftrightarrow \delta)\right\} \\
& \quad-\frac{1}{2}\left\{\Phi_{\alpha}^{* \mu \sigma} \Phi_{\beta}^{* \rho \nu}\left[H\left(\mu \nu ; \mu^{\prime} \nu^{\prime}\right)-\Delta(\mu \nu ; \lambda \tau) H\left(\lambda \tau ; \mu^{\prime} \nu^{\prime}\right)\right] \Phi_{\delta}^{\mu^{\prime} \nu^{\prime}} \Phi_{\gamma}^{\rho \sigma}+(\alpha \leftrightarrow \beta ; \gamma \leftrightarrow \delta)\right\} .
\end{aligned}
$$

If the $\Phi$ 's are chosen to be the eigenstates of the microscopic quark Hamiltonian, the intraexchange term $V_{m m}^{i n t}$ is cancelled (see Eq. (69). This cancelation is the main effect of the renormalization of the wave function, higher order terms in the expansion give small corrections. This is explicitly demonstrated in the following two examples.

The derivation within the Fock-Tani representation of the corrections discussed above is trivial, since these always appear in such a form that the microscopic Hamiltonian acts on a bound state kernel. With a little of experience with the manipulation of the equations of motion of the quark operators, the relevant terms in these equations can easily be identified. It is easy to convince oneself that the lowest order corrections for the effective mesonmeson potential come from terms in the microscopic quark-antiquark interaction of the form $q_{\mu}^{(1) \dagger} \bar{q}_{\nu}^{(0) \dagger} \bar{q}_{\rho}^{(0)} q_{\sigma}^{(5)}+$ h.c.. In order to obtain $q^{(5)}$ and $q^{(5) \dagger}$, the generator $F$ of the transformation at fourth order is needed.

It is not difficult to show that the fourth order $O$ operator for mesons is given by

$$
O_{\alpha}^{(4)}=\frac{3}{8} \Delta_{\alpha \beta} \Delta_{\beta \gamma} M_{\gamma}-\frac{1}{8} M_{\beta}^{\dagger}\left[\Delta_{\alpha \gamma}, \Delta_{\beta \delta}\right] M_{\delta} M_{\gamma}+\frac{1}{4} M_{\beta}^{\dagger}\left[M_{\alpha},\left[\Delta_{\beta \gamma}, M_{\delta}^{\dagger}\right]\right] M_{\gamma} M_{\delta},
$$

In the proof of the commutation relation of Eq. (40) up to fourth order, it is useful to make use of the Jacobi identity for bosonic operators $A, B$, and $C$,

$$
[A,[B, C]]+[C,[A, B]]+[B,[C, A]]=0
$$

Next, the fifth order quark equation of motion is obtained, and only the terms that are relevant for the lowest order orthogonality corrections are retained. These come with an antiquark creation operator $\bar{q}^{\dagger}$ and three ideal meson operators. This is because the 
$\bar{q}_{\rho}^{(0)}=\bar{q}_{\rho}$ must be canceled in the expression $q_{\mu}^{(1) \dagger} \bar{q}_{\nu}^{(0) \dagger} \bar{q}_{\rho}^{(0)} q_{\sigma}^{(5)}$, and since $q_{\mu}^{(1) \dagger} \bar{q}_{\nu}^{(0) \dagger} \sim m^{\dagger}$, extra three ideal meson operators are needed to form an effective meson-meson interaction.

The equation of motion for the quark operator to fifth order, retaining only the relevant terms for the orthogonality corrections, is given by

$$
\begin{aligned}
\left.\frac{d q_{\mu}^{(5)}(t)}{d t}\right|_{\text {relev }} & =-\delta_{\mu \mu^{\prime} 1} \frac{1}{2}\left[M_{\beta}^{(0)}(t) \Phi_{\alpha}^{\mu^{\prime} \nu} \bar{q}_{\nu}^{\dagger(0)}(t) \Delta_{\alpha \beta}^{(4)}(t) m_{\beta}^{(0)}(t)\right. \\
& \left.+\Delta\left(\mu^{\prime} \nu, \rho^{\prime} \sigma^{\prime}\right) \Phi_{\alpha}^{* \rho \sigma} \Phi_{\beta}^{\rho^{\prime} \sigma} \Phi_{\gamma}^{\rho \sigma^{\prime}} \bar{q}_{\nu}^{(0) \dagger}(t) M_{\alpha}^{(0) \dagger}(t) M_{\beta}^{(0)} m_{\gamma}^{(0)}(t)\right] .
\end{aligned}
$$

In the same way, a similar equation is obtained for $\bar{q}_{\mu}^{(5)}(t)$, which is necessary for the transformation of the kinetic energy operator. Integrating the equations and taking $t=-\pi / 2$, results in

$$
\begin{aligned}
& T(\mu) q_{\mu}^{\dagger(5)} q_{\mu}^{(1)}+T(\nu) \bar{q}_{\nu}^{\dagger(5)} \bar{q}_{\nu}^{(1)}+\left[V_{q \bar{q}}(\mu \nu ; \rho \sigma) q_{\mu}^{(5) \dagger} \bar{q}_{\nu}^{(0) \dagger} \bar{q}_{\sigma}^{(0)} q_{\rho}^{(5)}+\text { h.c. }\right] \\
& =+\frac{1}{2}\left\{\left[\Phi_{\alpha}^{* \mu \sigma} \Phi_{\beta}^{* \rho \nu} \Delta(\mu \nu ; \lambda \tau) H\left(\lambda \tau ; \mu^{\prime} \nu^{\prime}\right) \Phi_{\delta}^{\mu^{\prime} \nu^{\prime}} \Phi_{\gamma}^{\rho \sigma}+(\alpha \leftrightarrow \beta ; \gamma \leftrightarrow \delta)\right]\right. \\
& \left.+\left[\Phi_{\alpha}^{* \mu \nu} \Phi_{\beta}^{* \rho \sigma} H(\mu \nu ; \lambda \tau) \Delta\left(\lambda \tau ; \mu^{\prime} \nu^{\prime}\right) \Phi_{\delta}^{\mu^{\prime} \sigma} \Phi_{\gamma}^{\rho \nu^{\prime}}+(\alpha \leftrightarrow \beta ; \gamma \leftrightarrow \delta)\right]\right\} m_{\alpha}^{\dagger} m_{\beta}^{\dagger} m_{\delta} m_{\gamma} .
\end{aligned}
$$

Clearly, when the $\Phi$ 's are the eigenstates of $H$, this will lead to an expression that is equal to and the opposite sign of $V_{m m}^{i n t}$ in Eq. (69).

For the baryons, the exact same procedure is taken. The fourth order $O$ operator is given by

$$
O_{\alpha}^{(4)}=\frac{3}{8} \Delta_{\alpha \beta} \Delta_{\beta \gamma} B_{\gamma}-\frac{1}{8} B_{\beta}^{\dagger}\left[\Delta_{\alpha \gamma}, \Delta_{\beta \delta}\right] B_{\gamma} B_{\delta}+\frac{1}{4} B_{\beta}^{\dagger}\left\{B_{\alpha},\left[\Delta_{\beta \gamma}, B_{\delta}^{\dagger}\right]\right\} B_{\gamma} B_{\delta}
$$

The use of the Jacobi identity for fermionic operators $A, B$, and $C$,

$$
[A,\{B, C\}]+[C,\{A, B\}]+[B,\{C, A\}]=0
$$

is useful for demonstrating the anticommutation relation of Eq. (125). As in the case of mesons, the cancelation of the intra-exchange part of the effective baryon-baryon interaction is attained.

Next, the RGM is used with an exactly soluble model to demonstrate that the main effect of the orthogonality correction for the meson-meson effective interaction is the cancelation of the intra-exchange term. We consider the scattering of two mesons, where the quark and 
the antiquark have masses $m_{q}$, and use an harmonic potential for the microscopic interaction in Eq. (93), namely,

$$
U(\boldsymbol{p})=-\int \frac{d \boldsymbol{r}}{(2 \pi)^{3}} e^{-i \boldsymbol{p} \cdot \boldsymbol{r}}\left(C+\frac{1}{2} k \boldsymbol{r}^{2}\right)=\left(\frac{1}{2} k \boldsymbol{\nabla}_{\boldsymbol{p}}^{2}-C\right) \delta^{(3)}(\boldsymbol{p}),
$$

where $C$ is a constant which fixes the oscillator's ground state. For this interaction, the Fock-space amplitude $\Phi$ is given by Eq. (97), with $b^{2}=\sqrt{3 / 2 m_{q} k}$, and the total energy of a single meson is

$$
E(\boldsymbol{P})=\frac{\boldsymbol{P}^{2}}{4 m_{q}}+2 m_{q}+\frac{3}{m_{q} b^{2}}+\frac{4 C}{3} .
$$

The evaluation of normalization kernel and its square root can be done analytically. The results are,

$$
\begin{aligned}
& N(\alpha \beta ; \gamma \delta)=\delta^{(3)}\left(\boldsymbol{P}_{\alpha}-\boldsymbol{P}_{\gamma}\right) \delta^{(3)}\left(\boldsymbol{P}_{\beta}-\boldsymbol{P}_{\delta}\right)-\frac{1}{6} \mathcal{N}_{E}\left(\boldsymbol{P}_{\alpha} \boldsymbol{P}_{\beta} ; \boldsymbol{P}_{\gamma} \boldsymbol{P}_{\delta}\right) \\
& N^{-\frac{1}{2}}(\alpha \beta ; \gamma \delta)=\delta_{\alpha \gamma} \delta_{\beta \delta} \delta^{(3)}\left(\boldsymbol{P}_{\alpha}-\boldsymbol{P}_{\gamma}\right) \delta^{(3)}\left(\boldsymbol{P}_{\beta}-\boldsymbol{P}_{\delta}\right)+C_{N} \mathcal{N}_{E}\left(\boldsymbol{P}_{\alpha} \boldsymbol{P}_{\beta} ; \boldsymbol{P}_{\gamma} \boldsymbol{P}_{\delta}\right)
\end{aligned}
$$

where

$$
\mathcal{N}_{E}\left(\boldsymbol{P}_{\alpha} \boldsymbol{P}_{\beta} ; \boldsymbol{P}_{\gamma} \boldsymbol{P}_{\delta}\right)=\delta^{(3)}\left(\boldsymbol{P}_{\alpha}+\boldsymbol{P}_{\beta}-\boldsymbol{P}_{\gamma}-\boldsymbol{P}_{\delta}\right)\left(\frac{b^{2}}{2 \pi}\right)^{\frac{3}{2}} e^{-\frac{b^{2}}{4}\left[\boldsymbol{P}_{\alpha}^{2}+\frac{\boldsymbol{P}_{\gamma}^{2}}{2}+\frac{\boldsymbol{P}_{\delta}^{2}}{2}-\boldsymbol{P}_{\alpha} \cdot\left(\boldsymbol{P}_{\gamma}+\boldsymbol{P}_{\delta}\right)\right]} .
$$

with

$$
C_{N}=\frac{\omega}{2} \lim _{k \rightarrow \infty} \sum_{m=1}^{k}\left(\frac{\omega}{2}\right)^{m-1}\left(\prod_{n=1}^{m} \frac{2 n-1}{n}\right)
$$

where $\omega=1 / 6$. In obtaining the closed form for the square root $N^{-\frac{1}{2}}(\alpha \beta ; \gamma \delta)$, we used the remarkable property of the function $\mathcal{N}_{E}$, that $\left(\mathcal{N}_{E}\right)^{k}=\mathcal{N}_{E}$.

The partial sums $C(k)$,

$$
C(k)=\sum_{m=1}^{k}\left(\frac{\omega}{2}\right)^{m-1}\left(\prod_{n=1}^{m} \frac{2 n-1}{n}\right),
$$

are plotted in Figure 2 below. The important fact to notice in this figure is that the series is rapidly convergent, and that for $k \geq 2$, the values of the $C(k)$ 's have almost reached their asymptotic value, $C(\infty) \sim 1.145$. This means that the bulk of the effect of the orthogonality corrections can be accounted for by retaining only the first term in Eq. (177). 


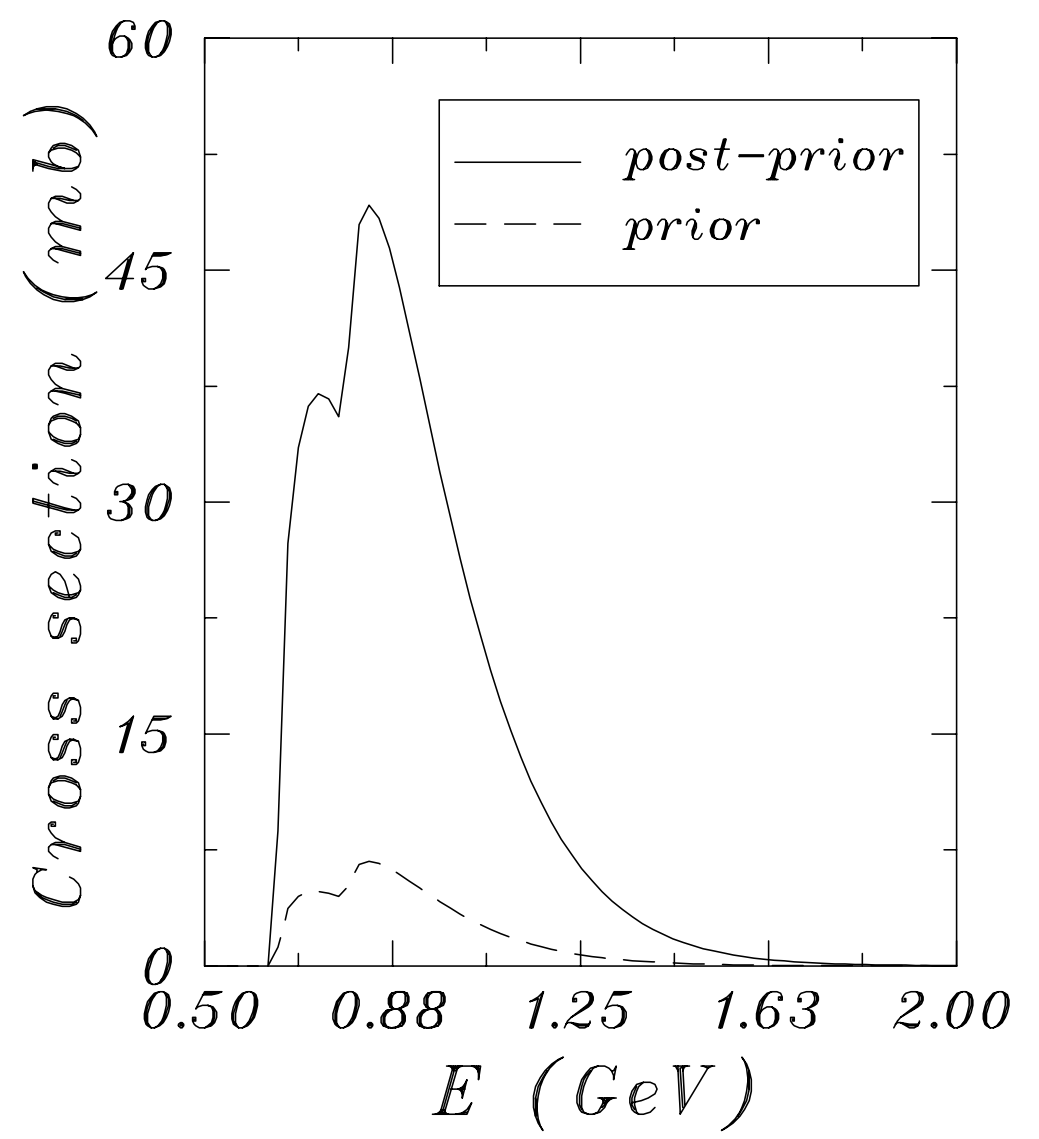

Figure 2. The partial sums $C(k)$ of Eq. (190).

The full RGM equation can be separated into two equations, one describing the free motion of the c.m. of the mesons, and the other describing their relative motion. Writing $\bar{\Psi}\left(\boldsymbol{P}_{\Lambda} ; \boldsymbol{p}\right)=\psi\left(\boldsymbol{P}_{\Lambda}\right) \varphi(\boldsymbol{p})$, where $\boldsymbol{P}_{\Lambda}$ and $\boldsymbol{p}$ are respectively the total c.m. and relative momenta of the two mesons, the equation for $\varphi(\boldsymbol{p})$ can be written as

$$
\int d \boldsymbol{p}^{\prime}\left[\frac{\boldsymbol{p}^{2}}{M} \delta^{(3)}\left(\boldsymbol{p}-\boldsymbol{p}^{\prime}\right)+\lambda \mathcal{V}\left(\boldsymbol{p}, \boldsymbol{p}^{\prime}\right)\right] \varphi\left(\boldsymbol{p}^{\prime}\right)=E_{r e l} \varphi(\boldsymbol{p}),
$$

where $E_{\text {rel }}=E_{\Lambda}-E_{c m}$, and the "potential" $\mathcal{V}\left(\boldsymbol{p}, \boldsymbol{p}^{\prime}\right)$, which is the result from the renormalization of the kinetic and potential ( $V^{i n t}$ and $V^{e x c}$ ) energies, is given by

$$
\mathcal{V}\left(\boldsymbol{p}, \boldsymbol{p}^{\prime}\right)=\left(\frac{b^{2}}{2 \pi}\right)^{\frac{3}{2}}\left[\frac{b}{4 m_{q}}-\frac{1}{24 m_{q}}\left(\boldsymbol{p}^{2}+\boldsymbol{p}^{\prime 2}\right)\right] e^{-\frac{1}{4}\left(\boldsymbol{p}^{2}+\boldsymbol{p}^{\prime 2}\right)},
$$

and $\lambda=C_{K}+C_{V}$, with

$$
C_{K}=6 \frac{2(1-\omega)^{\frac{1}{2}}+\omega-2}{(1-\omega)^{\frac{1}{2}}}, \quad C_{V}=\frac{1}{(1-\omega)^{\frac{1}{2}}} .
$$


Since $\omega=1 / 6, C_{V}+C_{K}=1.046$ is obtained. Had we used only the first two terms in the expansion of $N^{-1 / 2}$ in Eq. (177), we would have obtained $\lambda=1$, instead of $\lambda=1.046$. The effect of the higher order terms is, therefore, less than $5 \%$.

This result stands for the case of an harmonic oscillator potential and gaussian Fock-space amplitudes $\Phi$, but it seems reasonable to expect that for other potentials and amplitudes the situation will not be extremely different from the present one. Of course, a check of the rate of convergence of the expansion in Eq. (177) is advisable when other than gaussian functions are used for the $\Phi$ 's.

For the case of baryons, when using a gaussian form for the Fock-space amplitudes $\Psi$, the result is not different from the one for the mesons as described above. The net result of the higher order terms in the expansion in Eq. (177) is very small on the effective baryon-baryon potential. However, contrary to the case of the mesons, the exchange kernel for baryons does not have the property $\left(\mathcal{N}_{E}\right)^{k}=\mathcal{N}_{E}$. Nevertheless, there is an interesting approximation scheme that might be useful for future calculations, as we shall shortly describe.

The normalization kernel for baryons is given by,

$$
\begin{aligned}
N(\alpha \beta ; \gamma \delta) & =\delta_{\alpha \gamma} \delta_{\beta \delta}-N_{E}(\alpha \beta ; \gamma \delta)=\delta_{\alpha \gamma} \delta_{\beta \delta}-9 \Psi_{\alpha}^{* \mu_{1} \mu_{2} \mu_{3}} \Psi_{\beta}^{* \nu_{1} \nu_{2} \nu_{3}} \Psi_{\gamma}^{\nu_{1} \nu_{2} \mu_{3}} \Psi_{\delta}^{\mu_{1} \mu_{2} \nu_{3}} \\
& \equiv \delta_{\alpha \gamma} \delta_{\beta \delta}-\omega \mathcal{N}_{E}\left(\boldsymbol{p}_{\alpha} \boldsymbol{p}_{\beta} ; \boldsymbol{p}_{\gamma} \boldsymbol{p}_{\delta}\right)
\end{aligned}
$$

In the nucleon-nucleon case, $\omega$ is given by

$$
\omega \equiv \frac{3}{4}\left[1+\frac{1}{9} \boldsymbol{\tau}_{N}^{1} \cdot \boldsymbol{\tau}_{N}^{2}+\frac{1}{9}\left(1+\frac{25}{9} \boldsymbol{\tau}_{N}^{1} \cdot \boldsymbol{\tau}_{N}^{2}\right) \boldsymbol{\sigma}_{N}^{1} \cdot \boldsymbol{\sigma}_{N}^{2}\right]
$$

The approximation consists in factorizing the spatial part and summing the resulting series as

$$
\begin{aligned}
N^{-\frac{1}{2}} & =\left[1-N_{E}\right]^{-\frac{1}{2}}=1+\frac{1}{2} \omega \mathcal{N}_{E}+\frac{3}{8} \omega^{2} \mathcal{N}_{E}^{2}+\frac{15}{48} \omega^{3} \mathcal{N}_{E}^{3}+\ldots \\
& \approx 1+\mathcal{N}_{E}\left[\frac{1}{2} \omega+\frac{3}{8} \omega^{2}+\frac{15}{48} \omega^{3}+\ldots\right]=1+\varpi \mathcal{N}_{E}
\end{aligned}
$$

where $\varpi=-1+1 / \sqrt{1-\omega}$. When using a gaussian form for the amplitudes $\Psi$, two interesting facts were observed: first, there is a fast convergence of the exact and approximate series, and second, after the second term in the expansions, the approximate and exact results are practically indistinguishable from each other. Of course, as mentioned above, when other 
forms than a gaussian are used for the $\Psi$ 's, the rate of convergence of the expansion of the square root has to be checked.

\section{EXTENSION TO GENERAL FOCK-SPACE STATES}

In this section an extension of the Fock-Tani transformation to more general Fock-space states will be discussed. When dealing with quantum field theoretic quark models, the description of mesons and baryons might require the consideration of more general Fock states as those of a quark-antiquark pair and triplets of quarks. A meson state, for example, would be more likely to be of the form $\phi_{1}\left|q \bar{q}>+\phi_{2}\right| q \bar{q}(q \bar{q} q \bar{q})>+\cdots$ and certainly mixing with states containing gluons, such as $\mid q \bar{q} g>$, can be expected. Of course, methods can always be devised to take into account as much of such a mixing as possible into an effective Hamiltonian, and to avoid complicated Fock amplitudes. Nevertheless, one must be prepared to deal with more complicated Fock-space states as well.

The unitary transformation for such states is constructed with the same iterative procedure described in the previous sections, but the construction of the generator $F$ requires modifications. In the present section, the necessary modifications will be discussed through an example, and these will be expanded upon in a future publication [37] dedicated to the details of these derivations.

Consider a meson state of the form

$$
M_{\alpha}^{\dagger}=\Phi_{\alpha}^{\mu \nu} q_{\mu}^{\dagger} \bar{q}_{\nu}^{\dagger}+\Psi_{\alpha}^{\mu \nu \sigma} q_{\mu}^{\dagger} \bar{q}_{\nu}^{\dagger} g_{\sigma}^{\dagger}
$$

where $g_{\sigma}^{\dagger}\left(g_{\sigma}\right)$ is a gluon creation (annihilation) operator. The quark and antiquark operators satisfy the usual canonical anticommutation relations of Eq. (四). For the sake of simplicity, we assume the following canonical commutation relations

$$
\left[g_{\sigma}, g_{\sigma^{\prime}}^{\dagger}\right]=\delta_{\sigma \sigma^{\prime}}, \quad\left[g_{\sigma}, g_{\sigma^{\prime}}\right]=\left[g_{\sigma}^{\dagger}, g_{\sigma^{\prime}}^{\dagger}\right]=0 .
$$

Using these and Eq. (四), the following expression is obtained for the commutator of composite-meson operators

$$
\left[M_{\alpha}, M_{\alpha^{\prime}}^{\dagger}\right]=\delta_{\alpha \alpha^{\prime}}+C_{\alpha \alpha^{\prime}}
$$


where $C_{\alpha \alpha^{\prime}}$ contains, in addition to the $\Delta_{\alpha \beta}$ of Eq. (6), terms proportional to the amplitude $\Psi_{\alpha}^{\mu \nu \sigma}$ defined in Eq. (198). It is useful to decompose $C_{\alpha \alpha^{\prime}}$ as

$$
C_{\alpha \alpha^{\prime}}=C_{\alpha \alpha^{\prime}}^{0}+C_{\alpha \alpha^{\prime}}^{+}+C_{\alpha \alpha^{\prime}}^{-}
$$

$C_{\alpha \alpha^{\prime}}^{0}$ contains the usual $\Delta_{\alpha \beta}$ and operators with at least one annihilation operator on the right,

$$
\begin{gathered}
C_{\alpha \alpha^{\prime}}^{0}=-\Delta_{\alpha \beta}+\Psi_{\alpha}^{* \mu \nu \sigma} \Psi_{\alpha^{\prime}}^{\mu^{\prime} \nu^{\prime} \sigma} q_{\mu^{\prime}}^{\dagger} \bar{q}_{\nu^{\prime}}^{\dagger} \bar{q}_{\nu} q_{\mu}+\Psi_{\alpha}^{* \mu \nu \sigma} \Psi_{\alpha^{\prime}}^{\mu \nu \sigma^{\prime}} g_{\sigma^{\prime}}^{\dagger} g_{\sigma}-\Psi_{\alpha}^{* \mu \nu \sigma} \Psi_{\alpha^{\prime}}^{\mu^{\prime} \nu \sigma^{\prime}} q_{\mu^{\prime}}^{\dagger} g_{\sigma^{\prime}}^{\dagger} g_{\sigma} q_{\mu} \\
-\Psi_{\alpha}^{* \mu \nu \sigma} \Psi_{\alpha^{\prime}}^{\mu^{\prime} \nu \sigma} q_{\mu^{\prime}}^{\dagger} q_{\mu}-\Psi_{\alpha}^{* \mu \nu \sigma} \Psi_{\alpha^{\prime}}^{\mu \nu^{\prime} \sigma^{\prime}} \bar{q}_{\nu^{\prime}}^{\dagger} g_{\sigma^{\prime}}^{\dagger} g_{\sigma} \bar{q}_{\nu}-\Psi_{\alpha}^{* \mu \nu \sigma} \Psi_{\alpha^{\prime}}^{\mu \nu^{\prime} \sigma} \bar{q}_{\nu^{\prime}}^{\dagger} \bar{q}_{\nu} .
\end{gathered}
$$

$C_{\alpha \alpha^{\prime}}^{+}$and $C_{\alpha^{\prime} \alpha}^{-}$contain only terms that involve at least one $\Psi_{\alpha}^{\mu \nu \sigma}$,

$$
C_{\alpha \alpha^{\prime}}^{+}=\left[C_{\alpha^{\prime} \alpha}^{-}\right]^{\dagger}=\Phi_{\alpha}^{* \mu \nu} \Psi_{\alpha^{\prime}}^{\mu \nu \sigma} g_{\sigma}^{\dagger}-\Phi_{\alpha}^{* \mu \nu} \Psi_{\alpha^{\prime}}^{\mu^{\prime} \nu \sigma} g_{\sigma}^{\dagger} q_{\mu^{\prime}}^{\dagger} q_{\mu}-\Phi_{\alpha}^{* \mu \nu} \Psi_{\alpha^{\prime}}^{\mu \nu^{\prime} \sigma} g_{\sigma}^{\dagger} \bar{q}_{\nu^{\prime}}^{\dagger} \bar{q}_{\nu}
$$

It is not difficult to prove that the operator $O_{\alpha}$ that satisfies the commutation relations of Eq. (40) up to third order in the Fock amplitudes is given by

$$
O_{\alpha}=M_{\alpha}-\sum_{\alpha^{\prime}}\left(C_{\alpha \alpha^{\prime}}^{+}+\frac{1}{2} C_{\alpha \alpha^{\prime}}^{0}\right) M_{\alpha^{\prime}}+\sum_{\alpha^{\prime} \alpha^{\prime \prime}} M_{\alpha^{\prime}}^{\dagger}\left[M_{\alpha},\left(C_{\alpha^{\prime} \alpha^{\prime \prime}}^{-}+\frac{1}{2} C_{\alpha^{\prime} \alpha^{\prime \prime}}^{0}\right)\right] M_{\alpha^{\prime \prime}}
$$

The modification referred to above is that the components $C_{\alpha \alpha^{\prime}}^{0}, C_{\alpha \alpha^{\prime}}^{+}$and $C_{\alpha \alpha^{\prime}}^{-}$of $C_{\alpha \alpha^{\prime}}$ enter in a particular way into this expression. This is because the commutator of $O_{\alpha}$ with $M_{\beta}^{\dagger}$ must result in a Kronecker $\delta_{\alpha \beta}$, and the remaining operators of this commutator must annihilate the vacuum state, since $F M_{\alpha}^{\dagger}\left|0>=m_{\alpha}^{\dagger}\right| 0>$ is needed. Since $C_{\alpha \alpha^{\prime}}^{+}$does not annihilate the vacuum, the components of $C_{\alpha \alpha^{\prime}}$ must be combined such that they cancel out any operators which contain a creation operator on the right.

To conclude, we mention that the same iterative procedure outlined for the above example can be followed [37] for creation operators $A_{\alpha}^{\dagger}$ involving any number of quark, antiquark and gluon (or other bosonic) creation operators in the form

$$
\begin{aligned}
A_{\alpha}^{\dagger}= & \sum_{n_{q}=0}^{\infty} \sum_{n_{\bar{q}}=0}^{\infty} \sum_{n_{g}=0}^{\infty}\left(n_{q} ! n_{\bar{q}} ! n_{g} !\right)^{-1 / 2} \Phi_{\alpha}^{\mu_{1}, \ldots, \mu_{n_{q}}, \nu_{1}, \ldots, \nu_{n_{\bar{q}}}, \sigma_{1}, \ldots, \sigma_{n_{g}}} \\
& \times q_{\mu_{1}}^{\dagger} \cdots q_{\mu_{n_{q}}}^{\dagger} \bar{q}_{\nu_{1}}^{\dagger} \cdots \bar{q}_{\nu_{n_{\bar{q}}}}^{\dagger} g_{\sigma_{1}}^{\dagger} \cdots g_{\sigma_{n_{g}}}^{\dagger}
\end{aligned}
$$

where $n_{q}, n_{\bar{q}}$ and $n_{g}$ respectively represent the number of quarks, antiquarks and gluons. $\Phi_{\alpha}^{\mu_{1}, \ldots, \mu_{n_{q}}, \nu_{1}, \ldots, \nu_{n_{\bar{q}}}, \sigma_{1}, \ldots, \sigma_{n_{g}}}$ is the Fock-space amplitude, the index $\alpha$ represents the quantum 
numbers of the hadron and $\mu, \nu$ e $\sigma$ those of the constituents, as usual. Such an extension is particularly useful for treating meson-baryon couplings, and the derivation of an one-bosonexchange picture of the nucleon-nucleon force. Work is in progress in this direction.

\section{CONCLUSIONS}

In this paper, we have extended the Fock-Tani representation to hadronic physics. The formalism was used for a general class of constituent quark models, independently of a specific form of the microscopic interaction. We derived the unitary transformation iteratively as a power series in the Fock-space hadron amplitude, obtained the transformed quark and antiquark operators, and derived effective Hamiltonians. The effective Hamiltonians are hermitian and describe all possible interactions among the composite hadrons, and the interactions of the composite hadrons with their elementary constituents (quarks and antiquarks), consistent with the microscopic Hamiltonian. The transformed quark and antiquark operators are completely general, they depend only on the quark structure of the hadron states. Given a microscopic quark Hamiltonian, the effective Hamiltonians can be immediately derived. There is no restriction to relativistic or nonrelativistic kinematics. The method can be used with models where explicit gluon degrees of freedom (or other degrees of freedom such as Goldstone bosons) are present in the states and in the microscopic Hamiltonian, as well as with models of multicomponent Fock-space amplitudes.

The fact that in the Fock-Tani representation all field operators satisfy canonical commutation relations allows the direct use of the traditional field theoretic methods. For the baryon case, in particular, great opportunities for applications in many-baryon systems are envisioned. Different methods have been employed in the literature to study various aspects of quark degrees of freedom in nuclei. Since the traditional picture of the nucleus is that of a system of hadrons, the explicit dynamics of the color degree of freedom must be limited to very short distances. This means that an approach using quark degrees of freedom should minimally deviate from, as well as contain in some limit, the traditional one. In this sense, the effective Hamiltonian of the Fock-Tani representation has a well-defined limit, since it explicitly describes the interactions among hadrons; quark-quark and quark-hadron interactions are treated separately as "residual" interactions, since the effects of the bound states 
are explicitly subtracted from the microscopic interaction. Herewith the properties of the hadron-hadron interactions and nuclei at high densities and/or temperatures using quark degrees of freedom can be carried out in a systematic and controllable fashion.

\section{Acknowledgments}

The authors would like to express their gratitude to Professor Marvin Girardeau for his

help in clarifying several issues of the Fock-Tani representation. The authors acknowledge discussions with M. Betz, C. Maekawa and M.R. Robilotta. The present work was partially supported by the Alexander von Humboldt Foundation (Germany) and the Brazilian agencies CAPES, CNPq, FAPERGS and FAPESP. 


\section{REFERENCES}

[1] Y. Zhu, X. Wang, X. Zhao, F. Zeng, R. Hou, G. Wu, L. Teng, C. Yu, Y. He, and F. Wang, Comm. Theor. Phys. 7 (1987), 149.

[2] S. Pittel, J. Engel, J. Dukelsky, and P. Ring, Phys. Lett. B 247 (1990), 185.

[3] C. Wu and D.H. Feng, Ann. Phys. 135 (1981), 166; Comm. Theor. Phys. 1 (1982), 705; ibid 1 (1982), 811.

[4] F.J. Dyson, Phys. Rev. 102 (1956), 1217.

[5] J. Dukelsky and S. Pittel, Phys. Rev. C 45 (1992), 1871.

[6] S. Pittel, J.M. Arias, J. Dukelsky, and A. Frank, Phys. Rev. C 50 (1994), 423.

[7] A.I. Georgieva, R.P. Roussev, P.P. Raychev, M.V. Stoitsov, S. Pittel, and J. Dukelsky, Phys. Rev. C 52 (1995), 2131.

[8] M.V. Stoitsov, S. Pittel and J. Dukelsky, Phys. Rev. C 53 (1996), 3088.

[9] E.G. Nadjakov, J. Phys. G 16 (1990), 1473.

[10] J. Meyer, J. Math. Phys. 32 (1991), 2142.

[11] F. Catara and M. Sambataro, Nucl. Phys. A 535 (1991), 605.

[12] M. Reboiro and P. Ring, Paper 2.1.1 presented at the 1992 International Nuclear Physics Conference, Wiesbaden, Germany. Book of Abstracts.

[13] F. Catara and M. Sambataro, Phys. Rev. C 46 (1992), 754.

[14] M. Sambataro, Phys. Rev. C 52 (1995), 3378.

[15] M.D. Girardeau, Phys. Rev. Lett. 27 (1971), 1416.

[16] V.S. Vorob'ev and A.L. Khomkin,Teor. i Mat. Fiz. 8 (1971), 109 (in Russian). English translation: Consultants Bureau, Plenum Publ. Co, New York, 1972.

[17] M.D. Girardeau, J. Math. Phys. 16 (1975), 1901.

[18] For a list of references see Ref. [26]. 
[19] A. Klein and E.R. Marshalek, Rev. Mod. Phys. 63 (1991), 375.

[20] D. Bohm and D. Pines, Phys. Rev. 92 (1953), 609.

[21] S. Tani, Phys. Rev. 117 (1960), 252.

[22] See section 6. of: S. Weinberg, Physica A 96 (1979), 327.

[23] S. Weinberg, Phys. Rev. 130 (1963), 776; 131 (1963), 440; M. Scadron and S. Weinberg, Phys. Rev. 133 (1964), B1589; M. Scadron, S. Weinberg and J. Wright, Phys. Rev. 135 (1964), B202.

[24] M.D. Girardeau and J.D. Gilbert, Physica A 97 (1979), 42.

[25] J.C. Straton and M.D. Girardeau, Phys. Rev. A 40, (1989), 2991.

[26] C. Lo and M.D. Girardeau, Phys. Rev. A 41 (1990), 158.

[27] E.S. Swanson, Ann. Phys. (N.Y.) 220 (1992), 73; T. Barnes and E.S. Swanson, Phys. Rev. D 46 (1992), 131.

[28] D. Blaschke and G. Röpke, Phys Lett. B 299 (1993) 332.

[29] L. I. Schiff, Quantum Mechanics, McGraw-Hill, New York (1968).

[30] K. Martins, D. Blaschke, and E. Quack, Phys. Rev. C 51 (1995) 2723.

[31] D. Hadjimichef, G. Krein, S. Szpigel and J.S. da Veiga, Phys. Lett. B 367 (1996), 317.

[32] T. Barnes, S. Capstick, M.D. Kovarik and E.S. Swanson, Phys. Rev. C 48 (1993), 539.

[33] C.J. Joachin, Quantum Collision Theory, North Holland, Amsterdam, vol.2 (1983).

[34] G. Krein and S. Szpigel, in preparation.

[35] K. Holinde, Nucl. Phys. A 415 (1984), 477; G. Q. Liu, M. Swift, A. W. Thomas and K. Holinde, Nuclear Phys. A 556 (1993), 331.

[36] A. Faessler, A. Buchmann and Y. Yamauchi, Int. J. Mod. Phys. E 2, 39 (1993); M. Oka and K. Yazaki, in Quarks in Nuclei, edited by W. Weise (World Scientific, Singapore, 1989) p. 489. 
[37] M. Betz, D. Hadjimichef, M.D. Girardeau and G. Krein, in preparation; M.D. Girardeau, G. Krein and D. Hadjimichef, Mod. Phys. Lett. A 11 (1996) 1121. 